\title{
Abstracts of the Conference on Neuropsychological Rehabilitation, July 11-12, 2005, Galway, Ireland
}

\section{Conference hosted by:}

The National University of Ireland, the Psychological Society of Ireland and the British Psychological Society, Division of Neuropsychology.

\section{Organising Committee:}

Agnes Shiel, (Convenor), National University of Ireland, Galway, Ireland

Brian McGuire, Department of Psychology, National University of Ireland, Galway, Ireland

Barbara A. Wilson, Medical Research Council, Cognition and Brain Sciences Unit, Cambridge and the Oliver Zangwill Centre. UK

Robin Morris, Department of Psychology, Institute of Psychiatry, de Crespigny Park, London, UK Camilla Herbert, Brain Injury Rehabilitation Trust, Burgess Hill, West Sussex, UK Huw Williams, School of Psychology, University of Exeter, Exeter. UK

OPENING ADDRESS: Professor Barbara A. Wilson, OBE

\section{The Practice of Neuropsychological Rehabilitation}

Barbara A. Wilson and Sara Sopena

Medical Research Council Cognition and Brain Sciences Unit, Cambridge, UK and Oliver Zangwill Centre, Ely, UK

verview: Wilson's (2002) provisional model of rehabilitation synthesised
a number of different models that have influenced the field. The two
basic assumptions are that neuropsychological rehabilitation is concerned
with the amelioration of cognitive, social and emotional deficits caused by an
insult to the brain and the main purposes of such rehabilitation are to enable
people with disabilities to achieve their optimum level of well being, reduce
the impact of their problems on everyday life and help them return to their
own, most appropriate environments. From this it follows that no one model,
theory or framework can deal with all the difficulties facing people with brain
impairments. These often include multiple cognitive impairments as well as
accompanying social, emotional and behavioural problems. Methods: In
order to understand which parts of the synthesised model were most used by
practising psychologists engaged in neuropsychological rehabilitation, a
questionnaire was designed and sent to British and Australian psychologists.
Results: 45 people responded. Some of the main results are summarised. The
most frequently used treatment approaches derived from models of cognitive
behaviour therapy (67\%), cognitive neuropsychological theory (37\%) and
behavioural models ( $24 \%$ ). Psychodynamic models were only used by 3\% of
respondents. Most practitioners tried to reduce disabilities and handicaps 
rather than impairments. They made use of several strategies to do this, with the most frequent being compensatory techniques, making use of residual skills and restructuring the environment (between 90 and 100\% using these). Restorative approaches and attempts at anatomical reorganisation were rarely used. Conclusions: Practising psychologists in rehabilitation use a range of theoretical models and approaches in their clinical work confirming the view that we need a broad theoretical base when dealing with the complex problems faced by people with brain injury.

\title{
SESSION 1: ASSESSMENT ISSUES
}

\section{A Six-Year Retrospective Follow-Up Study of Neuropsychological Functioning in School Children Who Sustained a Head Injury Under Five Years of Age}

\author{
Phil Yates, ${ }^{1,2}$ W. Huw Williams, ${ }^{1}$ Ian Frampton ${ }^{1}$ and Anne Crum-Lindquist ${ }^{1,2}$ \\ ${ }^{1}$ School of Psychology, University of Exeter, UK \\ ${ }^{2}$ Royal Devon and Exeter Hospital, Exeter, UK
}

Qverview: The aim of this study was to determine if long-term effects in higher level cognitive functions, behaviour and academic performance were evident in late childhood as a result of head injury sustained under the age of five years. Methods: A representative sample of 14 children aged between 2 to 4 years, who attended an A\&E department as a result of a head injury, were followed up six years later in primary school. They were assessed on a battery of neuropsychological measures of attention, executive functions, and academic performance. Teacher and parent ratings of behaviour were also obtained. Comparisons were made with a control group of 23 non-headinjured children. Results: There were no statistically significant differences between the two groups on measures of academic performance and cognitive functioning. Significant differences were found on behavioural measures, particularly relating to parental reports of emotionality and difficulties with peer relationships. Fifty-three percent of head-injured children exhibited significant behavioural difficulties at six years post-injury when compared to $9 \%$ of healthy controls. Clinically significant difficulties were found for just below $10 \%$ of the head injury group on neuropsychological and academic measures. Some differences were found in scholastic performance, although no group performed below the average range. Conclusions: There are relatively few longitudinal studies following up children more than three years after head injury. This study indicates that at six years post-head injury behavioural problems are prevalent. No significant differences between head-injured children and controls were observed on measures of cognitive function and academic performance. Children with head injuries who attend A\&E may be at risk of developing behavioural difficulties in late childhood.

\section{Neuropsychological Profile and Adaptive Functioning in Children With Pervasive Developmental Disorders}

Sara Sopena, Barbara A. Wilson and Ayla Humphrey

MRC Cognition and Brain Sciences Unit, Cambridge, UK and Oliver Zangwill Centre, Ely, UK

Children with pervasive developmental disorders (PDD) who do not meet diagnostic criteria for autism and are not globally-learning disabled (IQ below 70) are generally placed in mainstream schools. These children require additional educational support because they are often reported to 
have problems staying on task, planning and organising their course work. The main purpose of this study was to determine whether these behavioural difficulties are associated with specific neuropsychological deficits, particularly executive and memory deficits. In addition, we also aimed to identify neuropsychological correlates of behaviour so that appropriate and specific interventions can be developed in order to remediate those behaviours that impede learning. Methods: Nineteen children with Pervasive Developmental Disorder - Not Otherwise Specified, Asperger disorder, and high-functioning autism were included in this study. All children completed a comprehensive neuropsychological battery for children (NEPSY) which evaluates a range of cognitive functions (attention/executive function, language, memory, sensorimotor and visuospatial abilities). Parents and teachers completed ecological valid questionnaires of executive function (Dysexecutive Questionnaire for Children-DEX-C and Behavioural Rating Inventory of Executive Function-BRIEF). Results: The results show that whereas children with PDD did not show significant impairment in any of the core domains measured with the NEPSY, both parents and teachers reported significant problems in executive function as measured with the DEX-C and the BRIEF. Conclusions: Behavioural measures of executive function are more indicative of daily and educational problems than a particular neuropsychological test in children with PDD. The results obtained from this study suggest that even if children with PDD have normal intellectual ability there is a need to provide specific educational support to compensate for their behavioural problems in order for them to access the curriculum in mainstream schools.

\title{
Head Injury Rates in a UK Prison Population: A Cause for Concern
}

Avril J. Mewse, Sarah Mills and W. Huw Williams

School of Psychology, University of Exeter, UK

There has been increased concern over the scarcity of provision of mental

1 health services for prison populations. This is in the context of many surveys of prison populations on mental health which has suggested that many such issues are common, and may be linked to social dislocation and, potentially, offending behaviour. There are very few studies that have examined the neurological status of people in prisons and resultant cognitive impairment. In this study we gave self-report questionnaires to prisoners in an UK prison. The survey included items on demographics, crime history, health status, mental health (depression, anxiety and PTSD) and head/brain injuries. Of the 453 approached, 198 responded - a 43\% return rate. 58\% reported events consistent with mild through to severe head injury. Data will be presented on associations between, type of injury, index criminal event, mood and health status. Implications for management of head injury in prison population and potential for rehabilitation will be discussed.

\section{Implicit Cognition is Impaired and Dissociable After Frontal Head-Injury}

\author{
L.A. Barker, J. Andrade, C.A.J. Romanowski, N. Morton and A. Wasti \\ Department of Psychology, University of Sheffield, UK
}

bjectives: The present study comprehensively assessed implicit cogni-
tion in frontally head-injured, CVA patients and matched controls.
Overview and Methods: Current theories propose that cognitive/
behavioural deficits shown by head-injured groups predominantly occur 
through executive dysfunction. Executive (top-down) processes initiate, inhibit or coordinate component (bottom-up) process and it is difficult to establish their separate contributions to behaviour. Recent studies showed deficits on a single implicit task after head injury (Beldarrain et al., 1999; 2002) suggesting that postmorbid behavioural change might reflect diminished bottom-up and top-down processes, separately or in concert. Researchers suggest that implicit cognition underpins the capacity to accurately 'read and respond' to social cues. This study examined implicit ability in 20 head-injured and nine stroke patients and age- and IQ-matched controls on four tasks; a serial-reaction time task (SRT), mere exposure effect task, automatic stereotype activation and hidden covariation tasks. Patients were assessed on a neuropsychological battery, and MRI scanned. Results: The head-injured group showed impaired implicit ability that dissociated across tasks. CVA patients and controls did not differ significantly. Patients impaired on implicit tasks had more cognitive deficits overall than those unimpaired, and a larger dysexecutive self/other discrepancy (DEX) score suggesting greater behavioural problems not attributable to size or laterality of lesion. Performance on one implicit task (SRT) correlated with a composite measure of executive function. Conclusions: Head injury produced executive dysfunction and impaired implicit cognition. Patterns of sparing and deficits suggest that bottom-up (implicit) and top-down (executive) processes may interact selectively, dissociate, and make separate contributions to behaviour. Future comparisons of implicit and explicit cognition should use several measures of each function, to ensure that they measure the latent variable of interest.

\title{
Patterns in the Neuropsychological Assessment of Culturally and Linguistically Diverse (CALD) Patients Across Two Settings in a Rehabilitation Hospital
}

\author{
Jamie Berry and Karen L. Wallace \\ Department of Clinical Psychology, St Joseph's Hospital Ltd, Sydney, Australia
}

\begin{abstract}
$O_{n}^{\prime}$ bjectives: The main purpose of this study was to investigate patterns of neuropsychological service provision for a heterogeneous culturally and linguistically diverse (CALD) population as compared to those from Anglo backgrounds. Methods: Retrospective analysis of consecutive neuropsychological assessment data for a 2-year period was undertaken. CALD patients were matched to non-CALD patients by diagnosis, age and level of education. The proportion of assessments, number of tests used, time spent conducting and preparing for neuropsychological assessment, and nature of tests used was compared for CALD vs. non-CALD patients across the settings of neurological rehabilitation (NR) and aged care rehabilitation/psychiatry (ACRP). Results: Fifty-nine of the 212 (28\%) neuropsychological assessments conducted were on CALD patients from 26 different backgrounds. Overall, significantly fewer tests were administered to CALD patients $(p<.01)$, particularly if an interpreter was present during the assessment $(p<.0001)$. Time spent assessing CALD patients was less than that for non-CALD patients $(p=.058)$. No difference in preparation time was found between the two groups. Significantly fewer verbal $(p<.003)$ and timed $(p<.009)$ tests were administered to CALD patients. The differences for number of tests administered, amount of time spent, and number of timed tests administered were significant for the ACRP population but not for the NR population. These populations differed significantly in age and education levels. Conclusions: This is one of the first studies to investigate neuropsychological testing
\end{abstract}


patterns in a rich heterogeneous sample of CALD rehabilitation patients. We found that use of an interpreter, type of referral question (e.g. diagnostic vs. descriptive), and age and education level of patients contribute to testing patterns with CALD patients. Clinical implications of these results are discussed in Wallace \& Berry (2005).

\title{
Development of Practical Clinical Guidelines for Neuropsychological Assessment of Heterogeneous Culturally and Linguistically Diverse (CALD) Populations in the Rehabilitation Setting
}

Karen L. Wallace and Jamie Berry

Department of Clinical Psychology, St Joseph's Hospital Ltd, Sydney, Australia

\begin{abstract}
bjectives: To develop guidelines for clinically relevant neuropsychological assessments of rehabilitation patients from heterogeneous CALD populations. Method: The first part of this paper summarises the literature pertaining to the neuropsychological assessment of CALD patients. Recent findings from a retrospective analysis of neuropsychological assessment of CALD patients from 26 different backgrounds $(n=59)$ (Berry \& Wallace, 2005) are compared and contrasted with previous findings in the literature. We provide clinical examples to illustrate some of the challenges inherent in working with such a diverse population. Results: Differences found between the existing literature and Berry and Wallace (2005) are purported to arise from the uniquely heterogeneous population in the latter study. Although it may be optimal to develop culture specific tests and norms for one or two major CALD groups (e.g., African and/or Hispanic Americans), this endeavour is unfeasible when working with smaller populations composed of multiple cultures and languages. Age, education level, use of an interpreter and type of rehabilitation setting (e.g., neurological vs. aged care) were found to influence the assessment process undertaken. Conclusions: Our study responded to a need to develop clinical guidelines for the neuropsychological assessment of diverse multicultural and multilingual communities. We advocate the use of process analysis rather than the development of additional test norms where the size of minority groups is prohibitive. This approach takes into account culture-specific variables, level of acculturation, demographic factors, psychosocial context, and affective and interpersonal functioning. The guidelines outlined in this study support a comprehensive tailored and ethical approach to assessing CALD rehabilitation patients.
\end{abstract}

\section{Neuropsychological and Psychiatric Consequences of Acute Encephalitis With Varying Aetiologies: A Case Series Analysis}

Stephen M. Pewter, Janice M. Kay, W. Huw Williams and Catherine Haslam School of Psychology, University of Exeter, UK

This study examines the ongoing cognitive and mental health implications 1 of acute encephalitis after the inflammatory phase of the illness has passed. A case series of 20 adults in the post-acute-phase of encephalitis was assessed using standardised measures of cognitive function, including memory, language and executive function, and their current psychiatric status was assessed using a 90-item symptom checklist (SCL 90). These results are discussed in relation to measures of insight, where patient's assessment of their own condition is compared to that of an additional rater 
using both the Dysexecutive Questionnaire (DEX) from the Behavioural Assessment of the Dysexecutive Syndrome (BADS). Results indicate a great deal of variation in cognitive impairment across the different aetiologies of encephalitis, with memory, naming and frontal-executive performance being differentially spared and damaged across viral subtypes. In contrast, severe psychiatric outcomes are common, with depression being particularly prevalent and the level of personal insight being a possible mediating variable. The implications with regards to the long term mental health and rehabilitation of encephalitis patients are discussed.

\title{
What's In This For Me? The Patient's Experience of Neuropsychological Assessment in the Context of Neurorehabilitation
}

\author{
Noelle Blake, ${ }^{1}$ Jan Burns ${ }^{2}$ and Martin Van Den Broek ${ }^{3}$ \\ ${ }^{1}$ Croydon Primary Care Trust, Carshalton, Surrey, UK \\ 2 Salomons Course in Clinical Psychology, UK \\ ${ }^{3}$ St George's Hospital, UK
}

bjectives: This paper will present the findings of a study that investigated the experience of neuropsychological assessment from the perspective of the patient with a neurorehabilitation setting. The study sought to gain an understanding of how patients viewed the purpose and relevance of the assessment process, particularly in terms of how it helped them understand their cognitive impairments and enhanced their sense of coping and self-esteem. Method: A within-group qualitative design was employed to investigate the experiences of nine men and women who presented for neuropsychological assessment in the context of an outpatient assessment clinic or as part of an evaluation for a rehabilitation programme. Verbatim transcripts of semi-structured interviews were analysed using Interpretative Phenomenological Analysis. Results: The results are discussed in the light of four master themes: expectations of assessment, the assessment encounter, the feedback process and the experience of illness/disability. The findings indicated that patients are poorly prepared for assessment but come with positive expectations for information that will help them understand and cope with their impairments. Their relationship with the psychologist is central in determining the quality of their experience and in facilitating improved self-esteem, coping and better awareness of cognitive strengths and deficits. The feedback process is haphazard and often fails to provide the patient with the information they seek in a timely and relevant manner and hence does not adequately capitalise on the educative and therapeutic opportunities inherent in the assessment encounter. Conclusions: It is concluded that neuropsychological assessment can have an educative and therapeutic function that should be further exploited in a diagnostic and rehabilitation context. The findings are considered in relation to the literature on sharing a diagnosis, coping with illness and the client-clinician relationship. The implications of the research findings for clinical training and neuropsychology service provision are discussed. 


\title{
SESSION 2: REHABILITATION ISSUES - COGNITIVE REHABILITATION
}

\section{Rightward Shifts in Visual Attention With Reduced Alertness: Is the Persistence of Unilateral Neglect an Exaggeration of a Normal Pattern?}

\author{
Tom Manly, Christopher Dodds, Melanie George and Veronika Dobler \\ MRC Cognition and Brain Sciences Unit, Cambridge, UK
}

\begin{abstract}
$O^{\prime}$ bjectives: Most patients with unilateral neglect recover from this severe spatial bias within days or weeks. Persistent forms of this debilitating condition are almost exclusively seen following right hemisphere damage and often co-occur with deficits in alertness/sustained attention. Observations, such as a reduction in neglect consequent upon pharmacological or auditory stimulation, have been argued to reflect a modulatory relationship between alertness and spatial attention. The specificity of this relationship is, however, difficult to test using only patient data in which attention to the left is originally deficient. The aim of the studies reported here was to examine changes in spatial bias in healthy participants as a consequence of naturally declining alertness. Methods: Adults from the healthy population performed a spatial task at two sessions. In one session they were sleep deprived in the other they were well rested. In addition, changes in performance across each session were examined. In the second study, a new group of healthy participants performed the rather tedious and repetitive spatial task over an hour. Results: Relative sleep deprivation was associated with a significant rightward shift in normal spatial attention. Subsequent analysis revealed that, even in the 'alert' session, a significant rightward shift occurred as a function of time-on-task. The second study replicated the timeon-task effect. Conclusions: It has been argued that the two hemispheres of the brain are engaged in a form of dynamic competition, each attempting to push attention into contra-lateral space. Conscious awareness of stimuli has been argued to involve the engagement of a 'general purpose' frontoparietal circuit in addition to activity in basic perceptual areas. Intrinsic alertness has been associated with predominantly right hemisphere activation within this circuit. This provides a mechanism whereby reductions in alertness may reduce conscious awareness of objects in left space and may reflect an analogous pattern to that seen in persistent unilateral neglect. Understanding recovery and persistence within conditions can lead to more appropriate targeting of rehabilitative and pharmacological interventions.
\end{abstract}

\section{Rehabilitation of Executive Functioning: A Controlled Cross-Over Study of an Attention and Problem-Solving Group Intervention}

\section{Eliane Miotto and Jonathan J. Evans \\ Gartnavel Hospital, University of Glasgow, UK}

$O$ bjectives: This study evaluated the efficacy of a group-based intervention aimed at improving executive functioning in patients with deficits in attention and problem solving arising from lesions of the frontal lobes. Method: Thirty patients with frontal lobe lesions were allocated to three groups, matching for age, IQ and aetiology. The three groups were (1) attention and problem-solving (APS) group, (2) information and education (IE) group, and (3) traditional rehabilitation (TR) group. Dependent variables examined included standardised measures of executive functioning, performance on a virtual planning task and a multiple errands test, and scores on self and others' ratings on a questionnaire measure of everyday executive 
functioning (DEX questionnaire). The group intervention was a 10-week group focused on (1) the development of strategies for managing attention and (2) learning to use a compensatory problem-solving framework to support problem solving and goal management in everyday life. Using a cross-over design, the two control groups participated in the APS intervention after completion of the first study phase. All groups were evaluated on four occasions, including a 6-month follow-up. Results: Analysis revealed that the intervention group improved in performance to a greater extent than the control groups on tests of executive functioning, and on the virtual planning and multiple errands tests. Ratings by others on the DEX questionnaire also improved significantly, though there was no difference in self-ratings. Each of the control groups showed improvement on measures of executive functioning only after participation in the intervention group. Conclusions: This study demonstrates that executive functioning in everyday life can be improved in people with acquired brain injury through the use of a group intervention focused on learning compensatory strategies.

\title{
In Search of an Ecologically Valid Measure of the Dysexecutive Syndrome: Can Virtual Reality Help in Rehabilitation?
}

\author{
Ashok Jansari, ${ }^{1}$ Sally-Anne Duncombe, ${ }^{1}$ Candy Wong ${ }^{1}$ and Lesley Murphy ${ }^{2}$ \\ ${ }^{1}$ School of Psychology, University of East London, UK \\ ${ }^{2}$ Rehab UK, London, UK
}

bjectives: The accurate assessment of individuals with dysexecutive syndrome (DS) is vital for their effective rehabilitation. Method: Jansari et al. (2004) created a realistic and ecologically-valid office-based role-playing task to tap the major deficits seen in the dysexecutive syndrome. As well as a realworld version, the task was also created to be run on a computer as a virtualreality (VR) environment. The findings showed that the task was able to significantly discriminate DS patients from matched controls (NCs) and that the VR findings replicated the real-world findings; this is important from a point of cost-effectiveness. The current study extends the findings of Jansari et al. (2004) by creating a second analogous environment. The overall aim is to allow the clinician to use one task as a baseline measure before rehabilitation, then the second to assess the effectiveness of particular rehabilitation regimes (e.g., Brainwave-R) and the generalisability of these treatments. Results: Results showed that the new task successfully discriminated the DS group from a NC group. Additionally, fine-grained analysis of individual patients' performance on specific cognitive constructs revealed impairments which had often been missed on formal clinical testing. Conclusions: The findings are discussed with reference to ecological-validity and clinical utility of experimental paradigms.

\section{Investigation of Auditory Stimulation of Autobiographical Memory in Alzheimer's Disease: Exploration of Potential Mechanisms}

\author{
Muireann Irish, ${ }^{1}$ C. Cunningham, ${ }^{1}$ J.B. Walsh, ${ }^{1}$ D. Coakley, ${ }^{1}$ B.A. Lawlor, ${ }^{1}$ I.H. Robertson ${ }^{2}$ \\ and R.F. Coen ${ }^{1}$ \\ ${ }^{1}$ Mercer's Institute for Research on Ageing, St. James's Hospital, Dublin, Ireland \\ 2 Department of Psychology, Trinity College Dublin, Dublin, Ireland
}

bjectives: Previous research demonstrated an enhancing effect of
music on autobiographical memory (ABM) in a small sample of mild 
to moderate Alzheimer patients (AD) (Foster \& Valentine, 2001) using a substantially modified adaptation of the MMSE. Potential mechanisms underlying this effect were posited, such as attention absorption, anxiety reduction and increased arousal. No attempt was made to investigate these mechanisms further. The aim of this research was to replicate the original study, using a more methodologically stringent design, to tease apart the potential contributory factors underlying this enhancing effect. Methods: Repeated measures experimental design with 2 groups; mild $\mathrm{AD}(n=10$, MMSE: > 17/30) and age, gender and education matched healthy controls ( $n=10$, MMSE: 25-30). Participants were tested on 2 occasions, one week apart, in music and silence conditions, with order counterbalanced across groups. The Autobiographical Memory Interview (AMI) was used to assess ABM recall. The State Trait Anxiety Interview (STAI) investigated changes in baseline and transitory anxiety levels. The Sustained Attention to Response Task (SART) was used as an index of attentional functioning, whilst the Galvanic Skin Response technique gauged fluctuations in tonic arousal. As per the original study, music used was the 'Spring' movement from Vivaldi's Four Seasons. Results: There was a significant improvement for patients' $\mathrm{ABM}$ recall in the music condition with an interaction for condition*group $(p<.005)$. There were no differences in terms of overall arousal, or errors during SART performance. A significant reduction in state anxiety compared with baseline levels was found in the music condition $(p<.001)$. Conclusions: This study has demonstrated a significant enhancing effect of background music on ABM recall in mild AD. Analysis of contributory factors suggests anxiety reduction in the music condition as the potential underlying mechanism.

\title{
SESSION 3: AWARENESS
}

\section{Vegetative State and Minimally Conscious State Survey: Comparative Analysis of the Views of Clinical Neuropsychologists and Speech and Language Therapists}

\author{
F. Colin Wilson', Jocelyn Harpur ${ }^{2}$, Nigel McConnell ${ }^{3}$ and Niall Pender ${ }^{4}$ \\ ${ }^{1}$ Joss Cardwell Centre, Green Park HealthCare Trust, Belfast, UK \\ ${ }^{2}$ Thompson House Hospital, Down Lisburn H\&SS Trust, Lisburn, UK \\ ${ }^{3}$ Fire Sert, University of Ulster Jordanstown (UUJ), Belfast, UK \\ ${ }^{4}$ Department of Clinical Psychology, Royal Hospital for Neurodisability, London, UK
}

ackground: Few studies have examined the clinical knowledge base, pro-
fessional training routes and opinions in relation to the management of
individuals with vegetative state or minimally conscious state relative to cur-
rent recognised model of best practice and care. Objectives: To evaluate the
understanding and opinions of clinical neuropsychologists and speech and lan-
guage therapists in relation to the assessment and management of vegetative
and minimally conscious patients relative to current practice guidelines.
Method: Semistructured postal surveys were sent to UK-based practitioner full
members of the Division of Neuropsychology (307) and speech and language
therapy managers (371). The questionnaire included information on postqual-
ification training, area of professional practice, assessment and management
practices, professional-family involvement and opinions on access to rehabil-
itation. Difference(s) in assessment and management practice among clinical
neuropsychologists and speech and language therapists were examined.
Result: Overall, $27 \%$ returned questionnaires ( 76 neuropsychologists and 101
speech and language therapists). Vegetative state and minimally conscious 
states were poorly defined by both clinical neuropsychologists and speech and language therapists. Less positive attitudes regarding the potential value of rehabilitation services, were reported by those who currently do not work with this patient group. Conclusions: Differences in definition, reported understanding and assessment of vegetative state and minimally conscious state were observed among both clinical neuropsychologists and speech and language therapists. The need to improve understanding and the use of appropriate standardised assessment procedures is highlighted. There is a dearth of specific assessment and management training in relation to vegetative and minimally conscious state patients.

\title{
The Role and Relevance of Self-Awareness in Achieving Rehabilitation Gains: Empirical Evidence and Case Illustrations
}

\author{
Tamara Ownsworth ${ }^{1}$ and Linda Clare ${ }^{2}$ \\ ${ }^{1}$ School of Health and Rehabilitation Sciences, University of Queensland, St Lucia, Australia \\ ${ }^{2}$ School of Psychology, University of Wales, Bangor, UK
}

\begin{abstract}
$\mathrm{T}$ here are divergent views concerning the degree to which self-awareness is necessary for rehabilitation gains. One perspective is that individuals with awareness deficits are less likely to participate in and benefit from rehabilitation and, thus, interventions should focus on enhancing individuals' awareness of their deficits. This conviction has spurred the development of a variety of awareness interventions. Other researchers argue, however, that rehabilitation gains can be made without explicit awareness through taskspecific learning and behavioural adaptation. Additionally, it has been suggested that any potential benefits of increased awareness might be counteracted by heightened emotional distress. Therefore, clearer guidelines concerning the role and relevance of awareness in rehabilitation are needed to assist clinical decision-making. Objectives: (1) to examine empirical evidence concerning the degree to which self-awareness is necessary for rehabilitation gains; and (2) to present case study evidence concerning individual responses to different rehabilitation approaches. Methods: A critical literature review and case study methodology. Results: The review identified a number of issues that require consideration when selecting an intervention approach for individuals with awareness deficits. Using illustrative case studies, the following issues will be discussed: (a) how awareness is conceptualised and determined; (b) the perceived impact of poor self-awareness and possible adverse effects of targeting awareness; (c) the relative influence of neuro-cognitive, psychological and socio-environmental factors underlying awareness deficits; (d) the clients' goals and expectations in the intervention compared to those of the therapists' and caregivers'; and (e) the scope, intensity and resources of the rehabilitation context. Conclusions: Clinical recommendations will be made for achieving rehabilitation gains for individuals with awareness deficits.
\end{abstract}




\title{
Awareness Deficits and Neuropsychological Impairment After Traumatic Brain Injury
}

F. O'Keeffe, ${ }^{1}$ P. Dockree, ${ }^{1}$ P. Moloney, ${ }^{1,2}$ S. Carton ${ }^{3}$ and I.H. Robertson ${ }^{1}$

${ }^{1}$ Trinity College Institute of Neuroscience \& Department of Psychology, National University of Ireland, Galway, Ireland

${ }^{2}$ National Rehabilitation Hospital, Dun Laoghaire, Co. Dublin, Ireland

\begin{abstract}
Qverview: A common difficulty following traumatic brain injury (TBI) is lack of ability to accurately assess physical, cognitive and behavioural deficits. Self-awareness and realistic self-appraisal have been recognised as significant factors in rehabilitation and long-term outcomes for TBI patients (Hart et al., 2004; Prigatano, 1999; Ben-Yishay et al., 1985). This study examines awareness of deficits and error-monitoring abilities and their link to neuropsychological abilities a clinical group of TBI patients and controls. Methods: 30 high-functioning TBI patients and 30 neurologically-healthy matched controls participated in this study. Methods of assessing insight included an awareness interview, discrepancy scores between self and other rated questionnaires of everyday functioning, error detection on a go/no-go sustained attention task, a dual action task and a naturalistic actions task and accuracy of participants' predictions on a number of neuropsychological tests. Electrodermal activity was also recorded. Results: Results indicate that the TBI patients were indeed significantly impaired in accurate self-monitoring compared to controls across a number of these measures. Error awareness measures also correlated significantly with impairment on neuropsychological tasks of executive function. Conclusions: These results are discussed with reference to aspects of assessment of insight, the theoretical links between neuropsychological functions and self-awareness and to potential implications for rehabilitation of such deficits in TBI patients.
\end{abstract}

\section{Revisiting Awareness Interventions Following Acquired Brain Injury: 'Insights' From the Family Context}

Giles Yeates, Karen Henwood, Fergus Gracey and Jonathan Evans

Oliver Zangwill Centre, Ely, UK

Djectives: A family-focused critique of traditional awareness interventions following acquired brain injury (provision of feedback, psychoeducation and individual psychotherapy), based on the findings of a qualitative study on family sense-making following ABI. Methods: 6 participants, 3 with acquired brain injury (ABI) and a respective relative, were interviewed using an open-ended qualitative interviewing paradigm. The participants with ABI were identified by clinicians as lacking awareness in one or more domain of functioning. Participants commonly shared anterior damage, and time post-injury ranged from 2 to 10 years. Relatives included two cohabiting mothers and a wife. Interview transcripts were analysed using qualitative discourse analysis, focusing on content, meaning within social context, and social action in talk. Results: The study's main findings add to previous literature in highlighting as a multidimensional family and wider social context in which awareness difficulties are situated, constituted and maintained. Three main dimensions of contextual influence were: identified gaps in the provision of ABI information by professionals, pre-injury contextual meanings used to understand the effects of ABI, limited what was then considered a meaningful account of change in families. Complexity was evident in the process of family sense-making, involving 
reciprocally-influential interpersonal and social processes such as a contesting of perspectives. These social processes were themselves related to preinjury influences. Conclusions: Traditional awareness interventions are seen as insensitive to contextual complexity. Innovations and future revisions are suggested, drawn from an integration of family therapy and neuropsychology. These include: formulating the specific effect of ABI psycho-education on each family, strategic consideration of who provides feedback and in what way, use of electronic feedback, attention to communicative processes, and externalising family disagreements in relation to family members.

\section{DAY 2}

KEYNOTE ADDRESS - CROSS-CULTURE ISSUES

\section{Neurorehabilitation of Non-Western Peoples: The Impact of Colonisation and Other Issues}

Professor Jenni A. Ogden

University of Auckland, New Zealand

Tsing the Aotearoa New Zealand situation as an example, where the indigenous Maori has to fit within the white western rehabilitation system, I will outline some of the issues that we, as a cross-cultural nation, are beginning to address, with the aim of improving rehabilitation outcomes for Maori and Pacific Island people. The ongoing repercussions of colonisation for Maori is a difficult issue for NZ health services, but many of the problems relevant to neurorehabilitation that NZ faces are common to any context where most of the neuropsychological theories, tests, and neurorehabilitation specialists come from the dominant western culture. The influence of politics can also not be underestimated and this, too, is probably universal. It can be an exciting (and ongoing) challenge finding out how different cultures can marry ideas and values to achieve better health outcomes. Within the psychological community in NZ perhaps the most important factor for change has been educating ourselves about Maori history and values, and coming to an understanding 'from the soul' about the effects of colonisation (or being a minority), on health outcomes. From this we are gradually realising that our sacred standardised tests may not always be the best way to assess neuropsychological functioning for every culture (especially if it is economically impossible to quickly develop culture-appropriate tests for each culture), and that our western approach to rehabilitation may need to be adapted or even changed in fundamental ways.

\section{SESSION 4: ENHANCING NEUROREHABILITATION} - CASE STUDIES IN REHABILITATION

\section{Balints Syndrome: A Single Case Study}

Maeve Nolan and Michelle Verdonck, on behalf of a multidisciplinary team Royal Hospital, Donnybrook, Ireland

bjectives: This paper outlines the presentation, assessment, subsequent multidisciplinary rehabilitation and ongoing progress of a 39 -year-old 
woman with Balints syndrome. Method: Balints syndrome is characterised by three main clinical signs: ocular apraxia, optic ataxia and simultanagnosia. RC experienced postnatal depression following the birth of her second child and was admitted to hospital with vascular migrainous-type headaches. She developed reversible posterior leukoencaphalopathy resulting in swelling and oedema in the occipital cortex and parietal lobe, massive vasospasm of the intracerebral vessels and subsequent bilateral occipital, parietal and frontal strokes. On discharge she was functionally blind and was diagnosed with reversible posterior encephalopathy and a partial Balints syndrome and referred for rehabilitation. RC spent 8 months as an inpatient in the National Rehabilitation Hospital, Dublin and completed a comprehensive programme of medical, occupational therapy, speech and psychological intervention. Her rehabilitation program included a detailed neuropsychological assessment, intensive visuoperceptual retraining including visual scanning, searching and writing exercises, training in compensatory strategies and a functional programme designed to provide rehabilitation in contexts meaningful to her. In addition she received relaxation training and regular psychotherapeutic input to assist her adjustment within her family context. Video footage of occupational therapy input is included. Results: While continuing to experience residual visual and cognitive deficits RC made significant gains resulting in her discharge from outpatient treatment in January 2005. Significant improvement was noted in terms of objective neuropsychological assessment, activities of daily living and reading ability. RC continues to attend for outpatient treatment and is also currently taking part in a pilot programme of Nova Vision, a computer-based visual rehabilitation programme in Columbia University Hospital, New York. Conclusion: A multidisciplinary, comprehensive approach to rehabilitation including cognitive, functional and psychotherapeutic elements is recommended for this complex and relatively rare neurological syndrome.

\title{
The Implementation of Memory Aids in Cognitive Rehabilitation: A Single-Case Study
}

\author{
Emma Berry
}

Addenbrooke's Hospital, Cambridge, UK

he cognitive rehabilitation of a 31 -year-old woman with moderate
memory impairment following an anterior communicating artery haemorrhage is described. Initial neuropsychological assessment 6 weeks after surgery showed mild executive dysfunction and significant impairment in retaining new information. An intensive neuropsychological rehabilitation program was set in place, which began 3 months post-injury, and aimed to improve the client's memory, meta-memory and executive functioning; reduce carer strain and to enhance problem-solving skills; and aid the client's activities of daily living and enable her to care for her 2-year-old son alone. The implementation of a range of comprehensive external memory aids and internal memory strategies was followed by problem-solving training, designed to exploit the client's intact procedural memory by teaching the client to use the memory aids effectively. Results showed rapid learning of the efficient use of memory strategies accompanied by simultaneous improvement in everyday functioning and a generalisation of strategies to new problems. At two months post-rehabilitation the client's husband felt able to leave his son alone with his wife and also showed significant reduction in levels of carer strain on formal testing. The findings highlight the benefits of a comprehensive external cueing 
system, along with teaching problem solving strategies to use such cues, for certain patients with significant anterograde memory impairment.

\title{
Can Cognitive Capacity Be Temporarily Enhanced Through Brief and Intensive Cognitive Stimulation? Implications for Neurorehabilitation
}

\author{
Robin E.A. Green, Le-Anh Ngo and Brenda Melo \\ Toronto Rehabilitation Institute, Toronto, Canada
}

\begin{abstract}
Background: Anecdotally, people report feeling mentally sharper after peri$\boldsymbol{B}_{\text {ods of very effortful cognitive processing (e.g., studying for exams). In a }}$ pilot study, we found empirical evidence for this phenomenal experience and we sought to replicate the finding in the present study. Objective: To demonstrate that cognitive capacity in healthy subjects can be enhanced by a period of intensive cognitive stimulation. Methods: An intervention and matched control group $(N=12$, each) underwent pre-intervention baseline neuropsychological (NP) assessment (clinical attention, working memory, speed of processing, memory tests). The intervention group then engaged in daily effortful 'homework' for two weeks (e.g., memorisation of poetry passages; speeded oral-mental arithmetic) while the control group performed 5 minutes of light reading daily. After the two weeks, follow-up NP assessments (same tests; alternate forms) were completed. Results: Both groups showed practice effects, but the intervention group showed significantly enhanced verbal working memory and verbal learning/memory, replicating our pilot results. As well, a significant slow-down was observed on timed tests in the intervention group, with a commensurate reduction in error rates relative to controls. Conclusions: The intervention group's cognitive capacity appeared to increase on working memory and verbal learning/memory tasks, consistent with anecdotal report and our pilot research. Interestingly, the intervention group spontaneously altered their strategic approach, adopting a slower rate of responding in favour of increased accuracy, but there was no evidence that strategies aided working memory or long-term memory performances. As the training/intervention tasks were quite different from the outcome measures, these findings offer preliminary evidence that cognitive capacity can be enhanced (at least temporarily) by a period of intense cognitive stimulation that demands very effortful processing. Mental effort, as distinct from mental exercise/practice, could be harnessed to enhance recovery after brain injury. Implications for neurorehabilitation will be discussed.
\end{abstract}

\section{SESSION 5: OUTCOMES}

\section{Do Beliefs About Rehabilitation Based Upon the Theory of Planned Behaviour Predict Engagement?}

Theresa Powell

School of Psychology, University of Birmingham, UK

Qverview: The theory of planned behaviour (TPB) conceptualises the cognitive processes that influence health behaviours. It has been used in numerous studies to predict intention to engage in certain health promoting behaviours, for example, smoking cessation and cardiac rehabilitation. Surprisingly, it has never been used to explore engagement in acquired brain injury $(\mathrm{ABI})$ rehabilitation. According to $\mathrm{TPB}$, intention is a function of three factors: the individual's expectation of what rehabilitation will do for 
them, their beliefs about whether important people would approve of their doing rehabilitation, and their beliefs about whether they would cope with the activities required for rehabilitation. This study therefore explores whether beliefs and attitudes towards rehabilitation based upon TPB can predict intention to engage in rehabilitation after ABI as well as actual engagement in rehabilitation. Methods: A self-report questionnaire was developed to measure treatment outcome beliefs, perceived barriers, subjective norm and control cognitions. Intention to engage in rehabilitation was measured using the Motivation for Traumatic Brain Injury Questionnaire. Clinicians provided ratings of patient's engagement in rehabilitation, which was used to measure actual behaviour. The scales were administered to 40 participants with acquired brain injury (mean 14 months post injury). Results: The new scale showed good internal reliability and regression analyses demonstrated that 'treatment outcome beliefs' and 'perceived barriers' were the best predictors of intention to engage in rehabilitation. Conclusion: This is the first study to demonstrate a link between beliefs about rehabilitation and engagement according to TPB. Results emphasise the importance of patients understanding the aim and philosophy of rehabilitation, that is, it requires active participation and is compensatory rather than 'curative'. Therapists should also look more closely at individual barriers to engaging in rehabilitation such as difficulty being around people with disability.

\title{
The Long-Term Effect of Post-Acute Neuropsychological Rehabilitation: A Controlled Follow-Up Study
}

\author{
Henriette Aaby Svendsen and Tom Teasdale \\ Centre for Rehabilitation of Brain Injury and Dept of Psychology, University of Copenhagen, Denmark
}

\begin{abstract}
$O$ bjectives: To compare long-term outcome in a group of adult patients with acquired brain injury who underwent post-acute, neuropsychological rehabilitation, with a matched non-rehabilitated group of patients. We hypothesise that the rehabilitation group will have generally better outcome. Methods: 36 brain-injured patients who completed a post-acute rehabilitation program, typically 2 to 3 years after injury, were compared with 18 braininjured patients, matched on demographic and injury-related characteristics, who did not undergo post-acute rehabilitation. At 14 to 22 years post-injury, all patients were interviewed concerning current psychosocial conditions. Additionally, questionnaires - European Brain Injury Questionnaire (EBIQ), WHO Quality of Life Questionnaire, Hospital Anxiety and Depression Scale (HADS) - were completed by the patient and a close relative. Results: The rehabilitation group rated themselves as having significantly fewer symptoms on the EBIQ scales of general complaints and depression as well being lower on the HADS depression scale and having a better physical quality of life. Eighty-one percent had some form of employment, in contrast to 59\% of the control group, a difference which approached significance $(p=.09)$. The relatives of the rehabilitation group reported a significantly lower level of anxiety and depression, as well as a higher degree of physical, psychological and environmental quality of life. Conclusions: Within the potential limitations of sample sizes and matching, and consistent with our hypothesis, post-acute neuropsychological rehabilitation would appear to have a positive effect, on both patients and their relatives, even 12 to 17 years post-trauma.
\end{abstract}




\title{
Comparison of Two Stroke Rehabilitation Units: Part of a European Comparative Study
}

\author{
Nadina B Lincoln ${ }^{1}$ and B. Smith ${ }^{1}$ on behalf of the CERISE group ${ }^{2}$ \\ ${ }^{1}$ Institute of Work, Health and Organisations, University of Nottingham, UK \\ 2 This abstract was developed within the framework of the 'Collaborative Evaluation of Rehabilitation \\ in Stroke across Europe (CERISE)', Quality of life-key action 6, 2001-2005, contract number \\ QLK6-CT-2001-00170 funded by the European Commission and Bundesamt für Bildung und \\ Wissenschaft (CH). This project was conducted by I. Baert (B), P. Berman (UK), H. Beyens (B), N. \\ Brinkmann (D), L. Connell (UK), E. Dejaeger (B), W. De Weerdt (B), L. De Wit (B), H. Feys (B), \\ W. Jenni (CH), J. Jurkat (D), H. Kamsteegt (B), C. Kaske (CH), M. Leys (B), N.B. Lincoln (UK), \\ F. Louckx (B), K. Putman (B), B. Schuback (CH), W. Schupp (D) and B. Smith (UK).
}

\begin{abstract}
$A$ ims: It has been well established that the outcome of rehabilitation is better for patients treated on a specialist stroke unit than in nonspecialist settings. However, the extent to which all stroke units have comparable outcomes is not clear. The aim of the study was to compare the outcome of stroke rehabilitation in two stroke units in two hospitals in Nottingham. Methods: As part of a European comparative study (CERISE), stroke patients admitted to two stroke units were recruited. Consecutively admitted patients with first stroke were screened for inclusion on standard measures. They were assessed on measures of motor ability and independence in activities of daily living on admission and discharge from the units and at 2, 4, and 6 months after stroke. They were assessed on measures of mood and quality of life at 2, 4 and 6 months after stroke. In addition participants were observed using a standardised time sampling schedule to determine the frequency of rehabilitation interventions. Results: Of the 135 participants recruited, 70 were admitted to unit $\mathrm{A}$ and 65 to Unit $\mathrm{B}$. The two groups were not significantly different on demographic variables. The outcome was compared between the two units. There were no significant differences in outcome on the Barthel, Extended ADL scale or Hospital Anxiety and Depression scale (M-W U $p=.12-.98)$ despite different organisational structures and staffing levels. In addition the relation between the amount of therapy input and outcomes will be examined. Conclusion: Comparison of the outcome of patients receiving rehabilitation on different stroke units suggests similar outcomes. This supports the notion that the differences between stroke units are less than the differences between stroke units and other rehabilitation wards.
\end{abstract}

\section{Outcomes of Intensive Neuropsychological Rehabilitation: The Relationship Between the Dysexecutive Questionnaire (DEX) and European Brain Injury Questionnaire and SMART Goals Attainment}

Andrew Bateman and the Oliver Zangwill Centre (OZC) Clinical Team (Susan Brentnall, Jon Evans, Donna Gartland, Fergus Gracey, Clare Keohane, Rachel Megoran, Kate Psaila, Barbara Wilson and Giles Yeates)

Oliver Zangwill Centre, Ely, UK

$O$ bjectives: Questionnaire and goal attainment data have been collected at entry and discharge (week 24) from rehabilitation at OZC. Self- and independent ratings of brain-injury-symptom frequencies were assessed using the European Brain Injury Questionnaire (EBIQ) and dysexecutive symptoms (DEX) checklist. Change in scores over the course of rehabilitation, and relationships between baseline questionnaire responses and goal attainment at discharge were examined. Methods: Goal attainment is rated at 
discharge by the interdisciplinary team as 'fully', 'partially', or 'not achieved' against criteria specified within the client-centred goals. Questionnaires are provided to the client for self-rating and to a carer/relative to provide an independent rater perspective. Results: Of 95 clients seen to date, $73 \%$ are male and $76 \%$ have sustained a traumatic brain injury. Of 676 goals set for the clients 50 (7\%) of these were not achieved. The remainder were fully $(47 \%)$ or partially $(45 \%)$ achieved. Changes in questionnaire scores were found to be significant. Self-rating EBIQ data at baseline was partitioned by quartiles, and frequency of goal attainment ratings was examined by Chi-square tests and visual inspection. High scores (i.e., high symptom ratings $>140$ ) on the EBIQ were not associated with higher rates of non-achievement of goals. In contrast, low scores $(<100$, indicating fewer problems) appear to be associated with a higher frequency of fully achieving goals. Conclusions: The data demonstrate that the rehabilitation programme is effective in terms of goal attainment and changes in questionnaire scores. Goals were attained by clients across the range of scores on standardised questionnaires. This may indicate that goals are set with the client that are achievable, appropriate, and realistic. Measurement of efficacy of rehabilitation is difficult because of uncertainty around selection of outcome measures, and lack of a control group for comparison. Nonetheless, these data provide a foundation for further analysis.

\title{
Can Poorer Outcome After Severe Brain Injury Be Predicted by Poorer Responsiveness in the Acute Recovery Period?
}

\author{
Agnes Shiel ${ }^{1}$ and Barbara A Wilson ${ }^{2}$ \\ ${ }^{1}$ National University of Ireland, Galway, Ireland \\ ${ }^{2}$ MRC Cognition and Brain Sciences Unit, Cambridge, UK
}

Doorer outcome in terms of patients remaining in the vegetative state or the minimally conscious state is decided upon many months after the initial insult. By the time this becomes clear, inappropriate rehabilitation or indeed no rehabilitation may have been offered. The aim of this study was to evaluate levels of responsiveness in the acute stages of recovery from severe traumatic brain injury related to overall outcome four years later. Methods: This was a follow-up study of patients recruited to a prospective observational study of severe head injury carried out four years later. Of the original 88 cases, 66 were contacted and 41 patients or their carers agreed to participate in a followup study. Of these, eight met the criteria for the vegetative state (VS) or minimally-conscious state (MCS). Data collected in the prospective study using the Wessex Head Injury Matrix were examined together with data from the outcome study to ascertain whether any behaviours having potential predictive value could be identified. Correlation, regression and Mann-Whitney tests were used to evaluate the data. Results: Time taken to achieve a number of early behaviours differed significantly between the VS/MCS group and the other groups. These were duration of coma $(p<.01)$; obeying command $(p=$ $.01)$; watching person in line of vision $(p<.01)$; looking at person giving attention $(p<.01)$ and focusing on person talking $(p<.05)$. Conclusions: These results suggest that certain behaviours observed in the acute recovery period after severe head injury may be predictive of poorer outcome. However, the results can only be considered to be speculative and further research to confirm or refute them is required. 


\title{
SESSION 6: SOCIO-EMOTIONAL FUNCTIONING
}

\section{Loss of Emotional Responsivity in People with TBI? A Psychophysiological Study}

\author{
Clare Saunders, Skye McDonald and Rick Richardson \\ School of Psychology, University of New South Wales, Sydney, Australia
}

\begin{abstract}
$O_{p}^{b}$ bjectives: Emotion-recognition disorders are increasingly reported in people with traumatic brain injury (TBI). So, too, are reports of blunted affect and insensitivity to others. This has suggested a common neural system underlying recognition of emotionally charged information and initial affective reactions to emotional stimuli. Some previous research has suggested a link between the two but is based upon subjective report of emotional experience. Subjective report is not always a reliable index. To extend this work we examined the emotional responsivity of adults with TBI to emotionally charged material using the 'startle' probe. Using this procedure, the extent to which participants 'startle' (i.e., the magnitude of their eye blink) in response to a sudden noise is measured. Normally, people startle less when viewing pleasant material (e.g., erotica) and more when faced with aversive stimuli (e.g., torture victims). These differences are taken as an index of their underlying emotional state. Methods: In order to examine this effect in people with TBI we examined their eyeblink in response to pleasant, unpleasant and neutral scenes. Subjective ratings of the affect and arousal elicited by the various pictures were also obtained. Results: TBI impaired the normal potentiation of startle to unpleasant pictures, but did not change the normal attenuation of startle to pleasant pictures. Further, subjective ratings indicated that TBI participants found unpleasant pictures less arousing than did controls, although they showed normal awareness that these pictures were indeed unpleasant. Conclusions: This evidence adds to a growing picture that TBI can diminish the capacity for empathic responses to emotional situations (especially negative situations) that may, in turn, deprive the individual of important social cues.
\end{abstract}

\section{Threat Appraisals and the Avoidance of Activities After TBI}

\author{
Gerard Riley \\ School of Psychology, University of Birmingham, Edgbaston, Birmingham, UK
}

bjectives: The study explored anticipatory threat appraisals and the consequent avoidance of activities after traumatic brain injury (TBI), in terms of their frequency and their relationship to psychosocial outcome. Methods: In this context, an anticipatory threat appraisal refers to the anticipation of negative consequences if one engages in a particular activity. Qualitative methods were used to generate a list of such appraisals, which was in turn used to devise a self-report questionnaire. This questionnaire was then used in a quantitative study involving 50 participants with a TBI. The study investigated the frequency of the appraisals and consequent avoidance in the sample, and the relationship of these two variables to emotional status (anxiety and depression), coping style and quality of life. Results: A varied list of threat appraisals was obtained. Threat appraisals and consequent avoidance were frequent in the sample. Males and those whose TBI resulted from an assault reported more avoidance. As predicted, avoidance was correlated with both emotional status and quality of life. Threat-related avoidance proved a stronger predictor of these outcome variables than a measure of avoidant coping style. Conclusions: A core aim of rehabilitation is to facilitate engagement in valued roles and 
activities. Threat appraisals and consequent avoidance may constitute a significant obstacle to this. Understanding the content and implications of these appraisals may enable therapists to provide more effective support to people with a TBI in dealing with this obstacle.

\section{Neuropsychological Assessment of Socioemotional Functioning in Children}

James Tonks, Huw Williams, Ian Frampton, Phil Yates and Alan Slater

School of Psychology, University of Exeter, UK

T asting emotional and social-communication deficits are common among Lchildren who have suffered a traumatic brain injury. Such deficits often have a delayed onset, occurring at around 10 years old. In this paper it is advocated that a proportion of reported deficits are a result of compromise to emotion-processing systems in the brain. These systems function in reading emotion in others from analysis of eyes, face expression, or emotional vocal prosody for example. Little is known about the development of these skills, and while it is clearly necessary to track the development of such abilities, assessment measures and normative data are currently unavailable to those working with children. Methods: In the current study, novel and adapted measures of emotion processing were used in requisite tasks of spontaneous socioemotional judgement, reading emotion from voices, eyes and faces. These were used, in conjunction with a battery of tests of cognitive function, in gathering normative data from 67 'healthy' children aged between 9 and 15 . Questionnaires were used as a measure of socio-emotional behaviour. Results: Findings indicate that emotion processing is a product of reliably measurable and dissociable skills, which operate independently from higher cognitive functioning skills in children, aged 9 to 15. Proportional relationships were found between proficient specific emotional judgement skills and socioemotional behaviour, indicating the centrality of such skills in functional interaction. Regarding the development of skills in reading emotions, a marked stage of improvement in facial expression processing, which occurred at 10 years old, was found to be proportionately based upon improved visual processing skills. A slight fluctuation in performance on the tasks was observed across this six-year period of development. Conclusions: In general, the findings show that it is possible to develop measures of socioemotional processing, which are sensitive to developmental nature of emotion processing systems, and can differentiate between potential selective impairments to subsystems, when considering children with brain injuries.

\section{Comprehension of Pragmatic Language and Mentalistic Actions After Frontal Lobe Lesions}

Shelley Channon, Andrea Rule, David Maudgil, Asa Pellijeff, Jessica Frankl and Colin Shieff

School of Psychology, University College, London, UK

bjectives: The interpretation of much of what people say and do is affected by the social context in which it takes place. Pragmatic language plays an important role in social interactions, as does the ability to interpret and comprehend the thoughts and feelings of people around us. Research to date has shown that processing non-literal meanings is often impaired after brain injury, despite intact ability to process syntactic and semantic aspects of language. There is also evidence that ability to understand others' mental 
states (theory of mind) is affected by brain injury. This study was designed to investigate the comprehension of sarcasm and theory of mind after focal lesions. Methods: Participants with focal lesions were compared with healthy control participants on story comprehension. The sarcasm comprehension task involved a series of brief scenarios, each ending either with a sarcastic remark or with a control literal remark. The action comprehension task also involved a series of brief scenarios, each ending either with a mentalistic action or with a control physical event. Results: Those with lesions to regions of the frontal lobes were impaired relative to the control group in the comprehension of both actions and pragmatic language. The results suggested that comprehension of mentalistic actions or remarks was influenced by both medial and lateral frontal lesions. Conclusions: There are likely to be multiple routes to impairment in interpreting what people do or say, underpinned by different brain regions. Potential constituent skills include mentalising, social knowledge and executive skills.

\title{
SESSION 7: VOCATIONAL, RECREATIONAL AND PERSONAL GOALS
}

\section{Enhancing Capacity to Consent to Sexual Relationships: A Case Series Study With People With Intellectual Disability}

\author{
Eileen Dukes ${ }^{1}$ and Brian McGuire ${ }^{2}$ \\ ${ }^{1}$ Department of Psychology, Kerry Parents \& Friends Association, Ireland \\ 2 Department of Psychology, National University of Ireland, Galway, Ireland
}

\begin{abstract}
bjectives: Many challenges exist for clinicians in assessing the capacity of people with cognitive impairment to understand sexual information and make decisions about sexual relationships. In this study, a modified sexeducation program was delivered twice weekly for 10 weeks on an individual basis to four adults with a moderate intellectual disability. The aim of the intervention was to enhance sexual knowledge, safety behaviours and capacity to make sexual decisions. Method: A single subject multiple baseline methodology was used. Each participant completed the Sexual Consent and Education Assessment (SCEA; Kennedy, 1993), a measure designed specifically to assess capacity in those with cognitive impairment. The SCEA was administered pre-intervention, on a weekly basis during intervention, postintervention and at 6-month follow-up. Results: The results showed an increase in knowledge scores of up to $75 \%$ and an increase in safety scores of up to $30 \%$. At follow-up, there was a slight reduction from postintervention levels, but scores remained significantly above the preintervention level. Interrater reliability of $91 \%$ was established with a second independent rater. Conclusion: This preliminary study points to a beneficial effect of individually-tailored, intensive sex education, leading to a greater capacity to make decisions of a sexual nature in people with cognitive impairment.
\end{abstract}

\section{Evaluation of the Clinical Effectiveness of a Brain Injury Vocational Rehabilitation Service}

\author{
Laura Moore, ${ }^{1}$ Ingrid Wall ${ }^{2}$ and Brian McGuire ${ }^{1}$ \\ ${ }^{1}$ Department of Psychology, National University of Ireland, Galway, Ireland \\ ${ }^{2}$ QUEST Brain Injury Service, Galway, Ireland
}

The clinical value of postacute, community-based rehabilitation for 
debate. This study examined the clinical effectiveness of 'Quest', the only vocational rehabilitation program for people living in the West of Ireland. We examined the extent to which participation in an intensive multi-disciplinary rehabilitation programme predicted improvements in community integration, occupational status and psychosocial adjustment following traumatic brain injury (TBI). A nonrandomised case control study was employed with 17 clients, with measures taken preintervention, postintervention and at 3-month follow-up. We also assessed client satisfaction with the programme using focus groups. Initial results point to improvements on several of the key outcome measures, providing preliminary evidence of efficacy for the rehabilitation program.

\title{
Recreation Participation as a Function of Employment at 23 Years Post-Trauma in a Rehabilitation Sample With Severe Traumatic Brain Injury
}

\author{
Cheryl A. Soo, ${ }^{1}$ Robyn L. Tate, ${ }^{1}$ G.A. (Tony) Broe, ${ }^{2}$ Adeline E. Hodgkinson ${ }^{3}$ \\ and Ian D. Cameron ${ }^{1}$ \\ ${ }^{1}$ Rehabilitation Studies Unit, Northern Clinical School, Faculty of Medicine, University of Sydney, Australia \\ ${ }^{2}$ Prince of Wales Medical Research Institute, Sydney, Australia \\ ${ }^{3}$ Brain Injury Rehabilitation Unit, Liverpool Hospital, Sydney, Australia.
}

bjectives: Occupational functioning after traumatic brain injury (TBI) is work (RTW). Other aspects of occupation, such as recreation, are particularly pertinent given the low RTW rates. This study analysed in detail the occupational activities in TBI subgroups that were and were not working. Methods: Sixty-nine severely injured survivors from a consecutive rehabilitation series were interviewed, along with a non-brain-damaged (NBD) comparison group of 20 siblings. The main measure was a semi-structured interview documenting, inter alia, spare time and recreational activities. These were classified into a number of categories, including active leisure (eg., sports, hobbies) and nonspecific activities (e.g., TV, walks). Comparisons were made among the NBD group and two TBI subgroups: working (including sheltered work) versus nonworking. There were no differences between the two TBI subgroups regarding duration of post-traumatic amnesia. Results: A significantly larger proportion of the NBD group (90\%) was in regular employment compared with the TBI group (28\%). Interactions among ways in which time was spent were complex, but in overall terms there were no differences between NBDWorking and TBI-Working groups for active leisure (78\% and 84\% respectively), although a significantly larger proportion of TBI-Working group had active pursuits compared to the TBI-Non-Working subgroup (58\%). One-way ANOVA showed differences among the NBD and TBI subgroups in level of self-esteem $(F=10.1, p<.001)$. Results of post-hoc $t$ tests indicated selfesteem was significantly higher in the NBD group compared to the TBIWorking subgroup, which in turn showed a trend to be higher than the TBI-Non-Working subgroup. Conclusions: These results highlight the added disadvantage experienced by the large TBI subgroup that does not RTW. They reinforce the important role of rehabilitation programs in preparing those who are unlikely to RTW with occupational alternatives, which may also assist in maintaining psychological wellbeing. 


\title{
CLOSING ADDRESS: Associate Professor Robyn Tate
}

\section{The Natural History of Recovery After Severe Traumatic Brain Injury: Changes in Functional Status Between 6 and 23 Years Posttrauma}

\author{
Robyn L. Tate, ${ }^{1}$ G.A. (Tony) Broe,${ }^{2}$ Adeline E. Hodgkinson, ${ }^{3}$ Ian D. Cameron ${ }^{1}$ \\ and Cheryl A. Soo ${ }^{1}$ \\ ${ }^{1}$ Rehabilitation Studies Unit, Northern Clinical School, Faculty of Medicine, University of Sydney, Australia \\ 2 Prince of Wales Medical Research Institute, Sydney, Australia \\ ${ }^{3}$ Brain Injury Rehabilitation Unit, Liverpool Hospital, Sydney, Australia
}

bjectives: The published literature on traumatic brain injury (TBI) suggests stability of outcome after the initial posttrauma years, yet few studies have examined the natural history of recovery over an extended period. This study documented recovery patterns over 23 years in a consecutive series of 100 patients with very severe TBI receiving their primary rehabilitation at a regionally-based unit. Methods: At the 23-year follow-up, $91 \%$ of the sample was traced: 69 people were interviewed and the remaining 22 had either died $(n=17)$ or declined participation $(n=5)$. Sixty-eight participated in both medium (6 years) and long-term (23 years) follow-up interviews. Measures included the Craig Handicap and Reporting Technique (CHART), Sydney Psychosocial Reintegration scale (SPRS) and a semi-structured interview. Results: Levels of recovery at 23 years post-trauma varied across CHART scales with median percent independence of $100 \%$ for physical, $97 \%$ for mobility, $82 \%$ for cognitive, $65 \%$ for social and $47 \%$ for occupation. For the group as a whole, psychosocial outcomes on the SPRS appeared remarkably similar at the medium and long-term follow-ups, with the respective percentages being $28 \%$ versus $24 \%$ with a 'good' level of functioning, $43 \%$ versus $50 \%$ with 'limited', and $29 \%$ versus $26 \%$ with a 'poor' level of functioning. Yet the group data hide the marked variability that occurred. Matched-pairs comparisons for the two time periods revealed that $63 \%$ of individuals showed significant change between assessment occasions: improvement in specific psychosocial domains occurred in $25 \%$, decline in $32 \%$, and admixtures in 6\%. Conclusions: These results demonstrate that psychosocial outcome in the longer term after TBI is not immutable, but rather is a dynamic process of ongoing adjustment. The clinical implications include the need for a lifelong rehabilitation perspective combined with regular review in order to identify those at risk of deterioration, as well as those whose participation can be enhanced. 


\title{
POSTER SESSION 1: ASSESSMENT
}

\section{PostconcussionSyndrome (PCS) Following Very Mild Head Injury}

\author{
Maeve Walkin, Teresa Burke and Jack Phillips
}

Beaumont Hospital, Dublin, Ireland

\begin{abstract}
$O_{s}^{b}$ bjective: The primary aim of this study is to determine the range and severity of persisting PCS in a cohort of individuals who attended A\&E following a very mild head injury (HI). A secondary purpose was to examine baseline rates of PCS and knowledge of PCS in the general population. Methods: A three-part study was undertaken. Part A examined the frequency and severity of persisting PCS in mild HI. Fifty mild HI patients were recruited and each completed a questionnaire detailing pre-accident PCS symptom frequency and severity, the functional status questionnaire and the multidimensional health locus of control questionnaire. These patients were then re-evaluated at 3 and 6 months post-injury in terms of current PCS status. Part B looked at frequency and severity of symptoms associated with PCS in the general population. Two hundred healthy control subjects were recruited and completed a PCS checklist referring to current symptoms of PCS, the functional status questionnaire and the multidimensional health locus of control questionnaire. Part C examined knowledge of PCS in the general population. Two hundred healthy control subjects were asked to list the symptoms they would expect an individual with mild head injury to exhibit at 3 and 6 months post injury. Results: Initial results revealed a high prevalence of some symptoms in the general population, and a high rate of PCS symptoms pre-injury in the HI population. Follow-up results in the HI population will be presented and results will be discussed in relation to each of the three parts of the study. Conclusions: Conclusions and recommendations will be made with regard to the medical management of mild $\mathrm{HI}$ and also in terms of medico-legal aspects of persisting symptoms following HI.
\end{abstract}

\section{Unlike Posterior Brain Areas Ventrolateral Prefrontal Cortex Is Only Active During Intentional Processing}

Anja Dove, Russell Epstein and Adrian M Owen

MRC Cognition and Brain Sciences Unit, Cambridge, UK

bjectives: This study investigates how activity in the ventrolateral prefrontal cortex (VLPFC) and in posterior brain areas is modulated by purposeful processing or 'intention', by the stimulus type, and by the nature of the task (encoding or retrieval). Methods: In an fMRI study faces, scenes and abstract paintings were presented with the instruction to remember the picture ('intentional encoding'), to decide, whether it has been presented previously ('intentional retrieval') or to just look at it for the first ('incidental encoding') or second time ('incidental re-viewing'). Results: In striking contrast to the pattern of activity in the posterior brain regions, the VLPFC was only active whenever the stimuli were viewed with a specific purpose or intention in mind (to remember it or to make a decision). In all other areas, 'just looking' or simply taking in information from the environment without any specific plan was sufficient to elicit significant activity, if a 'suitable' stimulus was presented. Passively viewing faces and scenes elicited strong activity in the 
fusiform face area (FFA) and parahippocampal place area (PPA), respectively. An area in the medial temporal lobe (MTL) was active during passively viewing any of the stimuli. Conclusions: Whilst intention had an effect on the VLPFC and the posterior brain areas, VLPFC was the only area which was active only in the intentional condition. This result is in line with the hypothesis that the VLPFC is involved in executive processes that operate on information available in posterior association cortical areas (Petrides, 1994). The relevance of this finding for rehabilitation will be discussed.

\title{
Our Experience With PASAT by Patients After TBI in Czech Republic
}

I. Gregorova

Army Rehabilitation Centre Slapy and Vlatavou 252 08, Czech Republic

bjective: The presentation summarises our experience with an attention test for measuring attention deficits in patients with TBI. Degree of head injury was from mild to severe. Method: Subjects were 55 people aged 17 to 59. Patients were assessed with the attention test, the Paced Auditory Addition Task (PASAT). The PASAT makes it possible to examine a separate attention capacity using the method of a specified auditory serial task of adding. This is a test of rapid calculation, when a respondent should add the most recently heard number to the second last number and respond aloud. The number is selected from a range 1-9 and they are given in a random sequence. The test includes four sets of 50 numbers, with speed of number presentation increased in the single series. Results: According to our expectation, poorer performance occurred in patients with severe degrees of head injury. The performance depended on location of damage. Finally, the performances were worst when the speed of the presentation was increased. Conclusion: Our experience with the PASAT in patients with TBI indicates that the instructions of this test are too complicated for some patients and the test is demanding even when contact with the patient is good. Nevertheless it is suggested that the PASAT is a good test to measure separate aspects of attention capacity.

\section{Is There a Need for Integrating Neuropsychology and Sports Science in Rugby?}

\author{
W. Huw Williams, Tim Genders, Simon Farrington and Anna Falconer \\ School of Psychology, University of Exeter, United Kingdom
}

$\mathrm{T}$ There is a high incidence of concussion in rugby. Repeat concussion is also prevalent, and is a matter of particular concern. Younger athletes may be at particular risk. There may well be neurological deficits and neuropsychological decrements associated with such injuries. Also, there is some debate over whether males and females are at equal or differential risk of having such injury or suffering consequences from such injury. Most players seek to return to play at a competitive level. Concussion needs close management so that the risk of sequelae are reduced and chances of sporting performance enhanced. It may be the case that there needs to be an integration of neuropsychological techniques and sports science for improving risk management and for enabling return to play. A study is described investigating long-term neuropsychological effects of concussion in rugby players, of both sexes, aged 18 to 25 . There are few such studies on the long-term effects of concussion and multiple concussions, particularly regarding repeat concussion and gender. The study consisted of recording 
signs and symptoms of concussion with the Rivermead Concussion scale and a battery of both pen and paper and computer based neuropsychological tests. Over 50 rugby players were tested, from a range of skill levels (club, local and university team levels). This paper will describe findings related to whether there were no/single/multiple concussions, and regarding any gender differences. Implications for neurorehabilitation in the context of sports, in particular rugby, are discussed.

\title{
A Normative Study of Socioemotional Functioning in Young Adolescents
}

\author{
Sarah E. Wall, W.H. Williams and I. Frampton \\ School of Psychology, University of Exeter, Exeter, UK
}

bjectives: The purpose of the current study was to standardise a measure of socioemotional functioning for use with young adolescents aged 11 to 14 years. Methods: A questionnaire has recently been developed (Bramham et al., in preparation) that effectively identified deficits in social and emotional functioning in adults with acquired brain injury (ABI) relative to controls. Additionally, it revealed that patients show a lack of insight into their deficits. The current study sought to gain normative data on socioemotional functioning in typically developing 11- to 14-year-olds recruited through local schools. Each adolescent and their parent/guardian completed parallel versions of the Social-Emotional Questionnaire (SEQ). Validating and comparative data was obtained from the Strengths and Difficulties Questionnaire (SDQ; self and parent versions) and the Dysexecutive Questionnaire for Children (DEX-C). Results: Responses were received from approximately 100 adolescents and their parent/guardian (response rate: 46\%). Factor analysis was used to explore the factor structure of the SEQ. The final factor solution was analysed in association with key demographic information, including adolescent gender and age, and parental level of education. Correlational analyses were conducted to explore associations between the SEQ and both the SDQ and DEX-C. Conclusions: Following the analysis of this normative sample and identification of the underlying factor structure of socioemotional functioning in typically developing young adolescents it is anticipated that the SEQ will be used with young adolescents who have a history of ABI, to identify deficits and highlight areas for intervention.

\section{Neuropsychological Performance Versus Workplace Performance: Ecological Validity Revisited}

\author{
Agnes Rappaport
}

Prince of Wales Hospital, Randwick, Australia

Veuropsychological assessment (N/P) is generally believed to assist vocational rehabilitation by identifying cognitive strengths and weaknesses. However, it is important to consider the relevance of N/P results when adequate workplace performance has already been demonstrated. This paper presents two cases where a review N/P assessment was requested for what the author considers is inappropriate reasons. The first case concerns A, a 33-year-old electrician who suffered hypoxic brain damage following an accidental drug overdose. Despite slowness in processing information and some memory difficulties, he successfully returned to work, initially under the supervision of another electrician. Problems with 'slowness' and 'poor initiation' were identified, but there were 'no technical difficulties'. When A 
applied to work night shifts, his manager requested a N/P review to 'clear him' for night work. The second case involves B, a 19-year-old male who was injured in a motor vehicle accident (MVA). N/P assessment at 7 months post MVA identified deficits with verbal fluency and memory. B had enrolled in a landscaping course when he was unexpectedly accepted for training to become a flight attendant. After extensive training, B passed all his exams. He was on a 'standby' list to work on overseas flights when an urgent N/P review was requested in regard to concerns about B's safety. In both of these cases, the requests for N/P review were declined. This presentation discusses the ecological validity of N/P assessment in regard to actual performance on the job and discusses possible underlying reasons for the request for reassessment.

\title{
Premorbid Intelligence: Development of a Demographically Based Method to Estimate IQ in the Spanish Population
}

\author{
Alvaro Bilbao \\ National Brain Injury Rehabilitation Centre, Madrid, Spain
}

Ttroduction: An important aim of neuropsychological rehabilitation is to identify impairment in higher cognitive or intellectual functioning. In order to achieve this task the assessment of premorbid intellectual ability becomes crucial. The estimation of premorbid cognitive functioning will allow us to assess level of cognitive deterioration but also the best cognitive functioning that can be expected in rehabilitation. Estimating premorbid cognitive ability is also crucial in forensic practice and for research purposes. Methods that allow the estimation of premorbid intelligence have been developed. Estimates derived from these methods are typically based on one or both of the following sources of information: (a) life history variables; (b) measures of current cognitive functioning that are highly correlated with intellectual functioning and that are relatively unaffected by brain damage. Objective: To present a method of estimating premorbid intelligence in the Spanish population. Methods: The method we present has been developed applying linear regression models to the standardisation sample of the WAIS-III in the Spanish population. The model is based exclusively on demographic and socio-educational variables, allowing estimation free of the influence of neurological lesion sequelae. Results: The efficiency of the method we present is similar to that of other methods widely used in other countries. Conclusions: This new formula for estimating premorbid intelligence is reliable and can be used to set treatment goals in neurorehabilitation.

\section{Coal Gas Poisoning: Single-Case Study}

Petr Kulistak

Army Rehabilitation Center, Dreyerova 642/17 Prague, Czech Republic

Doisoning from coal gas affects many functions of brain areas, especially resulting in memory deficit. In our anecdotal case, two young college students, a male and female, were exposed to equal doses of coal gas while they were asleep. Very severe impairment occurred in the male, without any impairment in the female. The young man remained on respiratory control in the acute stage for 1.5 months and clinically he had severe spastic quadruparesis. Upon HMPAO SPECT showed slight hypoperfusion of gray brain matter in the parietal lobes. Neuropsychological examination at the beginning 
of rehabilitation revealed retrograde amnesia of approximately six months. Assessment of global memory (WMS) was normal (MQ = 121); but he showed prolonged simple and complex reaction times in both the auditory and visual modalities - $($ RTA $=275 \mathrm{msec} ;$ RTV $=363 \mathrm{msec} ;$ RTDV $=$ $578 \mathrm{msec})$; visual searching also showed prolonged latency $(10.57 \mathrm{msec})$. Similarly, impaired performance was also found on tests of executive functions (WCST, Stroop), spatial memory (Corsi blocks, ROCF) and global assessment of intellectual abilities (WAIS-R: FIQ = 102, VIQ = 124, PIQ = 78). After six months of rehabilitation, which included computer-assisted exercises in areas of cognitive functions, individual neuropsychotherapy, and occupational therapy, he attained normal levels of the impaired cognitive functions and test results. Additionally, we had available six year-old IST examination of our patient from high school, with a very superior result (Amthauer IQ = 146). At this time, his test results are in the average range $(I Q=105)$, with selected areas of decline (practical numerical ability, spatial and surface imagination). We monitor him neuropsychologically and with rCBF SPECT control.

\title{
Implicit Memory Assessment and Its Implication for Rehabilitation
}

Sara Sopena, Bonnie Kate Dewar, Eve Greenfield and Barbara Wilson

MRC Cognition and Brain Sciences Unit, Cambridge, UK

bjectives: We plan to develop a reliable and standardised test of implicit memory that can be used to assess people with memory deficits and help in the planning of a rehabilitation program. Methods: 180 normal controls and 50 people with memory deficits ranging in severity will be included in this study. To test implicit memory ability a perceptual priming task and a word completion task will be administered to both groups. All participants will also perform an explicit memory test (RBMT-E). Performance on both tests will be compared for both groups. Results: The study will enable us to obtain standardised norms for non-injured controls and patients with memory deficits on an implicit memory task. It is hypothesised that the control group will show intact explicit and implicit memory abilities whereas the patient group will show impaired explicit memory but intact or relatively intact implicit memory ability. Conclusions: Implicit memory is presumed to be the most preserved memory system in patients with memory deficits, including patients with traumatic brain injury, Alzheimer's or Parkinson's disease, or schizophrenia. Many rehabilitation programmes train new material through accessing information via the implicit memory system. However, implicit memory ability is not currently assessed with reliable and valid methods. There is a need to develop a standardised test of implicit memory that can give reliable information regarding intact implicit skills so that clinicians can incorporate such information into their rehabilitation programs.

\section{The Rivermead Perceptual Assessment Battery: Is It a Useful Tool for Occupational Therapists?}

\author{
Aoife Bagnall, Olga Hill, Margaret McGrath and Laura Parkes
}

ccupational therapists working in neurological rehabilitation have recognised the impact of perceptual skills on ability to perform functional tasks (Neistadt, 1988). For this reason occupational therapists working with patients with stroke or brain injury have an interest in identifying perceptual deficits 
early in assessment sessions so that they can plan to incorporate relevant intervention strategies. The RPAB is known to be one of the few standardised assessments predominantly designed for occupational therapists (Matthey et al., 1993). It is used to assess visual perceptual deficits in people suffering from head injury or stroke. This paper explores some of the statistical background behind the RPAB and considers the reliability, validity and clinical usefulness of the assessment.

\title{
S-100, Posttraumatic Amnesia and Neuropsychological Function Following Severe Traumatic Brain Injury
}

Sharon Watt, Arthur Shores and lan Baguely

Macquarie University, Sydney, Australia

\begin{abstract}
The relationship between blood serum concentrations of protein S-100
and neuropsychological functioning during the acute stages of severe traumatic brain $(N=23)$ were examined. Blood samples were taken within 12 hours of injury, and daily, up to 7 days post-injury. Within two weeks of emerging from post-traumatic amnesia (PTA), participants completed a battery of neuropsychological measures. These results were compared with a matched sample of healthy controls. S-100 obtained within the first 12 hours of injury not only reflected overall brain injury severity, but also related to neuropsychological deficits, with higher serum concentrations associated with poorer performance across most cognitive domains. PTA duration, measured by the Westmead PTA scale was found to be the strongest predictor of S-100 concentration $\left(\mathrm{R}^{2}=0.59\right)$. These findings suggest that measurement of serum protein S-100 may further aid in the rehabilitation process in the identification of individuals with severe TBI who are likely to experience cognitive difficulties.
\end{abstract}

\section{Tuberculosis Meningitis and Attention Deficit Hyperactivity Disorder in Children}

John Wait, L. Stanton and J.F. Schoeman

Department of Psychology, Stellenbosch University, South Africa

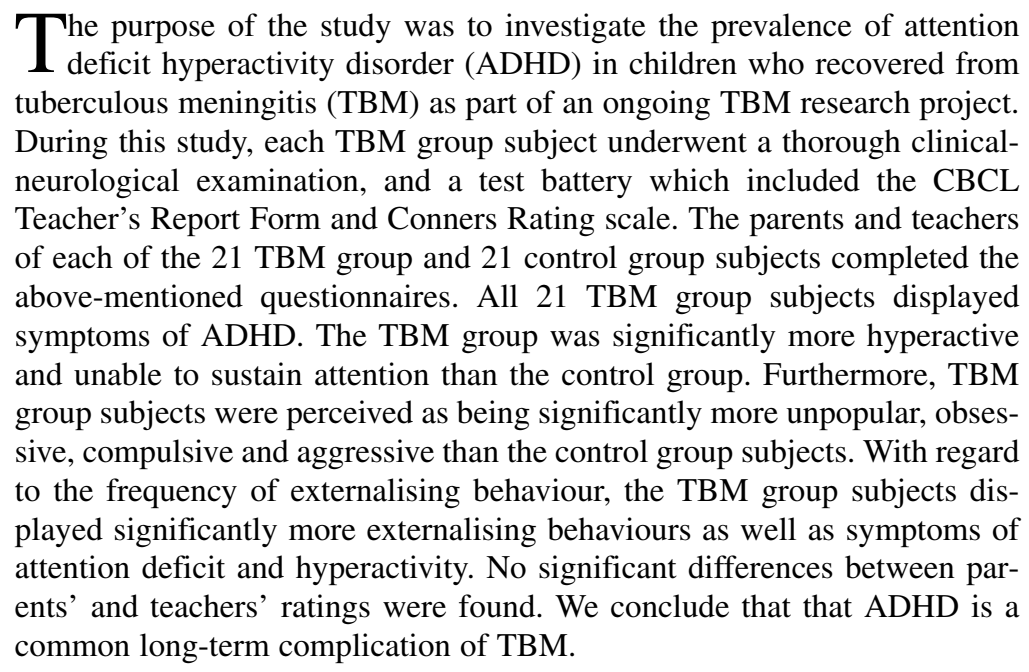




\title{
POSTER SESSION 2: REHABILITATION
}

\section{The Executive Group: \\ Group Treatment in the Management of Traumatic Brain Injury}

\author{
Valerie Twomey and Suzanna Doole \\ National Rehabilitation Hospital, Dublin, Ireland
}

The Executive Group is a multidisciplinary-led cognitive rehabilitation objectives of the group were to provide education and support and to facilitate awareness and generalisation of strategies learned. Six patients with ABI met group criteria and four committed to participating in the Executive Group. Ages ranged from 19 to $55 \mathrm{yrs}$ and all presented with mild cognitivecommunication impairments and were already engaged in inpatient rehabilitation. Assessments used to screen patients included the Rivermead Behavioural Memory Test and the La Trobe Communication Questionnaire. Evaluation of the effectiveness of the intervention included: the Working Memory Index scale of the Wechsler Adult Intelligence scale, The European Brain Injury Questionnaire and the Attention and Executive Function Rating scales. The group consisted of 6 weekly sessions addressing different topics following a set format. Each session was facilitated by 2 therapists and lasted for 1.5 hours. Results indicate overall increase in awareness and functioning in the areas of cognition communication and behaviour. Patients grasped basic knowledge of brain function. More importantly however they demonstrated an understanding of how their specific injuries resulted in behavioural, communicative, cognitive and physical symptoms. There was a decrease in the level of cognitive impairment reported by patients following the group intervention. This was observed functionally with increased use of compensatory strategies at the time, which was maintained at 6-month follow-up. The success of the group led to the introduction of further initiatives by the multidisciplinary team: Brainworks, a standard educational group; the Memory Group, an education and training group aimed to teach patients practical ways of coping with their memory difficulties.

\section{Effects of Self-Alert Training (SAT) on Sustained Attention Performance in Adult ADHD}

Redmond G. O'Connell, Mark A. Bellgrove, Paul Dockree and lan H. Robertson

Department of Psychology and Institute of Neuroscience, Trinity College, Dublin, Ireland

Drevious research has demonstrated that sustained attention deficits can be P temporarily ameliorated by the presentation of periodic auditory alerts designed to trigger increased top-down control of behaviour. In this study we developed a training protocol - Self-Alert Training (SAT) — in which participants learn to produce self-generated increases in alertness first in response to an auditory cue and later in response to an internally generated cue. The use of an alerting technique that is not reliant on particular environmental conditions can provide a highly flexible means of triggering controlled behaviour that is potentially applicable to a range of real-world settings. During SAT, visual feedback conveying the magnitude of each self alert event is provided via online changes in electrodermal activity (EDA). A group of adults with ADHD and a group of control participants were randomly assigned to the treatment condition or a video game practice control session each lasting 30 minutes. Efficacy of the treatment, relative to the 
control condition, is assessed via changes in reaction times and errors committed on the Sustained Attention to Response Task (SART). All participants completed four blocks of the SART prior to and following the treatment and control phases. EDA indices of alertness and performance monitoring during SART performance provided a further indication of treatment efficacy. Results are discussed in terms of the efficacy of SAT training for improving the sustained attention deficits of ADHD.

\title{
Identifying Frequent Antecedents Leading to Problem Behaviours in a Population of Acquired Brain Injury Clients
}

\author{
Catherine Mac Guinness \\ Crotched Mountain Adult Brain Injury Centre, New Hampshire, USA
}

$6 \mathrm{~T}$ is estimated that 2,000,000 Americans become victims of brain injury

leach year', (Burke, 1996). The aim of this project was to identify antecedents, which occurred frequently at an adult brain injury centre, resulting in problem behaviours on the units. Also this project introduced intervention strategies that could be used by direct care staff, to reduce the occurrence of identified antecedents and thus reduce the number of problem behaviours. A qualitative analysis was conducted at Crotched Mountain Adult Brain Injury Centre, Greenfield, New Hampshire, U.S.A. Data was collected from written incident reports (November 2003-April 2004), direct observation on the units ( 2 weeks, April 2004) and therapy team input. The principals of Applied Behavioural Analysis were used to identify antecedents leading to problem behaviours and to outline intervention strategies available to direct care staff. Follow-up analysis of written incident reports (April 2004-November 2004) was also conducted. Thirteen problem behaviours were identified and defined. The most frequent problem behaviours were physical abuse, verbal abuse, yelling and refusing. Twentyeight antecedents were identified. The most frequently occurring antecedents were depression, staff interactions, learned helplessness, physical and cognitive demands placed on clients and another client. Within the project, focus was shifted to the following overt and controllable antecedents, staff interactions, physical and cognitive demands, another client and family members. Seven intervention strategies were outlined with a focus on the method of Gentle Teaching. The findings of this qualitative analysis enabled the directors of the brain injury centre to focus on more comprehensive staff training and development to help reduce the problem behaviours on the units, leading to a more beneficial therapeutic environment for clients.

\section{Finding out if 'The Me Will Shut Down': Successful Cognitive Therapy of Panic with Neurological Symptoms - A Single Case Report}

Fergus Gracey, Paul Oldham and Rudi Kritzinger

Oliver Zangwill Centre, Ely, UK

Subarachnoid haemorrhage (SAH) and cavernoma are associated with Sincreased risk of seizure disorder and mood disturbance. There is mixed evidence for the efficacy of cognitive and behavioural therapies for anxiety disorders in acquired brain injury and seizure disorders. Development of evidence is complicated by the aetiology and presentation of emotional disorders in neurological conditions. This paper describes work with our client, 
Paul, who presented with panic symptoms in the context of seizures, migraine and other neurological symptoms. Objective: The assessment, therapy, and outcome of a cognitive approach to panic disorder in the context of SAH and cavernoma and related neurological symptoms is presented. Method: Multidisciplinary clinical assessment, including cognitivebehavioural assessment according to the model of Clark (1986) was carried out. A 10-session program of cognitive therapy was delivered with 2 followup sessions. Outcome was measured through goal attainment, changes in belief ratings of target cognitions and completion of standardised questionnaire measures. Results: The client attained goals of being able to go to the local supermarket, complete a long car journey and attend busy social gatherings without marked symptoms. Avoidance and other unhelpful coping behaviour was eliminated. Changes in identified target cognitions were also evident. Gains in these areas were maintained at follow-up. Self-rating of anxiety level was reduced at treatment end, with a slight increase at followup. Conclusion: Multidisciplinary assessment to identify possible anxiety symptoms in the context of neurological problems is warranted. Cognitivebehavioural therapy may offer a means of treating anxiety disorders where symptom presentation is complicated by neurological problems. Further investigation of the relationship between development of anxiety disorders, occurrence of neurological events, and processes of cognitive therapy following acquired brain injury is required.

\title{
Evaluation of a One-Off ‘Memory Group’ Intervention
}

\author{
Andy J. Champion, L. Higbed and A. Thomson. \\ Health Psychology Department, Gloucestershire Royal Hospital, UK
}

\begin{abstract}
A series of modules relating to the management of cognitive difficulties after acquired brain injury has been produced, with the intention of providing sufficient resources for any member of a multidisciplinary team to facilitate the sessions. This pilot study aims to evaluate one module, which relates to managing memory difficulties. Methods: Participants represented a range of aetiologies including stroke, traumatic brain injury and progressive neurological conditions. Pre-and post-intervention self-report measures of anxiety and depression (Hospital Anxiety and Depression scale), memory lapses (Everyday Memory Questionnaire) and use of memory strategies were taken. The intervention consists of a one-off session lasting approximately two hours, of which the first section provides adequate information about memory to underpin the second section, which covers a range of external and internal memory management strategies. Results: Results collected at the time of abstract submission relate to attendees at one group session ( $n$ $=5$ ). Three of the five attendees reported increased use of memory strategies, and increased use of memory strategies was associated with fewer reported memory lapses (mean EMQ score reduced by 19 points) and reduced psychological distress (mean HADS score reduced by 2.5 points). Data collection is ongoing (three sessions run to date) to allow more meaningful conclusions to be drawn. Conclusions: Use of the resources included in this one-off group intervention (including slides, handouts and script) aimed at people experiencing memory difficulties and their family members can increase the use of memory strategies and reduce the impact of memory difficulties on daily life. As the material is arranged in such a way as to be accessible to all members of the multidisciplinary team, it may represent a useful resource for inpatient and outpatient rehabilitation.
\end{abstract}




\title{
Using a Paging System in the Rehabilitation of an Encephalitic Patient
}

\author{
Hazel Emslie, Bonnie-Kate Dewar and Barbara Wilson \\ MRC Cognition and Brain Sciences Unit, Cambridge, UK
}

\begin{abstract}
$\mathrm{M}^{-1}$ emory and executive problems following encephalitis are common yet there are few published papers on the successful rehabilitation of such patients. We recently demonstrated (Wilson, Emslie et al., 2001, 2005) that a paging system could reduce the everyday memory and planning problems for people with nonprogressive brain injury. The 143 patients who participated in the study, comprised several diagnostic groups, including encephalitis. We report the case of a 49-year-old man, RC, who was 3 years post-encephalitis and was one of the participants in the 2001 study. A randomised control crossover design randomly allocated people to group A (pager first) or group B (waiting list first). Each participant chose his own tasks for which he needed reminders. During a two-week baseline, successful task achievement was documented. The group as a whole achieved a little over $47.14 \%$ of tasks but RC's task success was only $2.2 \%$. RC, who had been randomly allocated to group A, then received a pager for 7 weeks. During the last two weeks of this 7-week period, task achievement was documented again. RC now achieved 79.54 per cent of tasks which compared favourably with the $71.8 \%$ average achieved by the rest of group A. Group A participants then returned their pagers (which were passed to group B) and entered the last 7-week phase in the study . During the last two weeks of this stage, participants were monitored once more. RC failed to achieve any of his target tasks, returning to baseline levels. This was far below the average achievement of the group which was $67.23 \%$ of targets and indicates that he would need the paging system on a long-term basis.
\end{abstract}

\section{Memory Rehabilitation: Evaluation of a Group Intervention}

Valerie Twomey and Alison McCann

National Rehabilitation Hospital, Dun Laoghaire, Ireland

\begin{abstract}
$\mathrm{T}$ The NRH 'Memory Group' is an ongoing initiative designed to provide adults with acquired brain injury a controlled and supported environment within which to explore the nature, extent and remediation of their memory deficits. This present study evaluated the efficacy of a 5-week multidisciplinary therapy group. The group, designed by Neuropsychology and Occupational Therapy, included assessment, psycho-education, ecologically valid strategy teaching and generalisation training. Patients were selected on the basis of having a newly acquired memory deficit and were all inpatients in the NRH neurorehabilitation program. Preliminary results derived from pre-and post-measures of memory status and function indicate that group participants demonstrated a significant increase in overall shortterm memory scores on the Rivermead Behavioural Memory Test, the Prospective Memory scale and informal measures of memory-aid use. Participants also reported significant increase in their level of deficit awareness, which they report impacted on their motivation and participation in general rehabilitation. Training within the group setting enhanced participants' commitment to their rehabilitation. The group provided a secure and supported environment within which to explore possible remediation strategies. Further developments include the setting up of an alternative group for high-level memory/executive deficits, and delivery of the 'group' to outpatient programs.
\end{abstract}




\title{
Errorless Learning Versus Vanishing Cues: Which is the Most Effective Method?
}

\author{
Kathryn I. Hodder and Catherine Haslam
}

School of Psychology, University of Exeter, UK

\begin{abstract}
$O$ bjectives: The current study compared the effectiveness of errorless learning and vanishing cues for learning object-name associations in healthy adults, both under standard learning and dual task conditions - the latter to simulate patient performance. Methods: Participants were asked to learn the association between novel objects ('Greebles') and common surnames in four controlled conditions. These were trial-and-error learning, and one of the two memory-rehabilitation techniques (errorless learning or vanishing cues), each under standard and dual task conditions. The dual task condition was imposed in an attempt to reduce the memory performance of healthy adults to make it comparable with that of memory-impaired patients. Results: Results will help to confirm the advantage of errorless learning and vanishing cues techniques over trial-and-error learning. Furthermore, the direct comparison of errorless learning and vanishing cues under controlled conditions will allow us to determine which of these techniques is most effective in facilitating memory performance. Conclusions: If both errorless learning and vanishing cues prove to be effective methods of learning, this suggests that combining the techniques might lead to enhanced effectiveness compared to the individual methods. However, if one technique proves more effective, there may be little benefit from combining the techniques. These outcomes will make an important contribution to the evidence-base relevant to memory rehabilitation techniques and, in doing so, will allow clinicians to make more informed judgements about the intervention of choice.
\end{abstract}

\section{Training Face/Name Associations in Prosopagnosia}

\author{
Bonnie-Kate Dewar and Barbara Wilson \\ MRC Cognition and Brain Sciences Unit, Cambridge, UK
}

Qjective: Learning of face/name associations in CR, a woman with prosopagnosia secondary to herpes simplex encephalitis, was investigated in the present study. Methods: Ten months post injury, CR continues to report difficulty recognising familiar people. Formal assessment indicated impairment of face recognition and semantic knowledge of famous people, with preserved perceptual processing of faces. To establish the training protocol, three faces were selected - a man, a woman and child - and paired with fictitious names. Based upon the method described in Francis et al., (2002) CR was asked to change the name of the person into an image and link with this with a prominent feature of the photograph. Learning was consolidated via expanded rehearsal, with criterion of accurate recall after 5 minutes. Training was initially attempted with one face. Following carryover across sessions, training was attempted with the other two faces. Recall was tested ten minutes after completion of training. Results: CR was able to learn the face name association for one photograph ('Joe Marsh') and recall this two weeks later. Learning of the other two faces was also successful ('Sarah Little' and 'Nick Watkins') although learning of the third face required additional consolidation. However, CR was able to recall the three names when shown the photographs at test. Conclusions: Previous attempts to retrain face and person information in prosopagnosia have proved difficult. Using the errorless learning principles of improved learning when mistakes are prevented, we have shown intact learning of face/name associations 
in a woman with prosopagnosia. This will form the basis of a training protocol for retraining of familiar people, such as her immediate family. Issues such as incorporation of semantic information and techniques to improve generalisation will be discussed.

\title{
Time-of-Day and Learning in Younger and Older Adults
}

Michael Hogan

Department of Psychology, National University of Ireland, Galway, Ireland

\begin{abstract}
We investigated the effects of time-of-day on associative learning and memory performance of younger and older adults. We hypothesised that nonoptimal testing times would be associated with increased intra-individual variability and reduction in the benefits of practice during associative learning. Results indicated that older adults showed an increase in RT SD relative to mean RT in the evening. However, time-of-day did not have a significant effect on rate of learning or total learning. Mean RT was a better predictor of higher-level memory performance than was RT $S D$ when using data acquired in the morning; RT $S D$ was the better predictor when using evening data. Results are discussed in light of theoretical models of aging and learning, and implications for cognitive rehabilitation will be explored.
\end{abstract}

\section{Treatment of Phantom Limb Pain Using Motor Imagery}

Laura McAvinue and lan Robertson

Department of Psychology, Trinity College, Dublin, Ireland

bjective: A large proportion of amputees suffer pain in their missing limb, a condition known as phantom limb pain. This pain is notoriously difficult to treat. Here, a single case study was used to investigate the effects of motor imagery, or imagined movement of the body, on phantom limb pain. Methods: A patient, who had received a lower-limb amputation less than two years prior to the study and who reported a more-or-less constant level of low intensity phantom limb pain, participated in a 12-week treatment program. Throughout the program, intensity and quality of phantom limb pain were tracked by means of a diary, which the participant filled out three times daily. The program consisted of a 2-week training free baseline, followed by 4 weeks of general motor imagery training, 4 weeks of motor imagery exercises directed towards the phantom limb and a 2-week followup period. Training consisted of a 1-hour long training session each week and 10 minutes of homework each night in between sessions. Results: Selfreport of the patient and examination of the graphs of phantom limb pain indicated that the frequency of phantom limb pain was reduced in second phase of the motor imagery training (i.e., imagined exercises directed towards the phantom). Time series analysis is to be performed on the data. Conclusions: The results suggest that motor imagery might be an effective treatment for phantom limb pain. 


\section{POSTER SESSION 3: PERSONAL AND VOCATIONAL}

\section{Return to Work Following Moderate-Severe Traumatic Brain Injury}

Sarah McCrimmon

Cardiff and Vale NHS Trust, UK

bjectives: This study aimed to investigate differences in cognitive, behavioural and emotional factors and 'illness perceptions' of people who have $(n=20)$ and have not $(n=13)$ returned to work (RTW) following a moderate to severe traumatic brain injury. The study also aimed to investigate the subjective experiences of employed and unemployed persons in the context of returning and not returning to work. Methods: The study employed a cross sectional within and between group's design consisting of quantitative and qualitative measures. Independent samples $t$ tests were used to compare groups on all measures and a content analysis of the written responses on the interview schedule was conducted. Results: The unemployed group reported significantly higher levels of depression, fatigue and cognitive/behavioural/ mood difficulties. They perceived the consequences of the injury to be more severe, felt less in control over the course of the injury and resultant symptoms and held more negative emotional representations about the injury. A significantly higher proportion of this group were seeking compensation. No significant differences were obtained on measures of cognitive functioning. Content analysis indicated a lack of awareness of potential means of support, a prevalence of cognitive problems and fatigue for both groups and qualitative information on difficulties experienced within the workplace and coping strategies utilised. Conclusions: Content analysis indicated a need for teaching cognitive strategies within the workplace, for access to vocational rehabilitation/meaningful activity prior to RTW, for education of government schemes supporting RTW and ongoing emotional support on RTW. The study highlights the potential use of the 'illness perception questionnaire' in this population to promote understanding of people's perceptions of brain injury and facilitating a cognitive-behavioural approach to help challenge these perceptions. Forming a more positive representation of the injury may increase a person's likelihood of RTW.

\section{Life Goals of People Attending Neuropsychological Outpatient Assessment After Acquired Brain Injury}

Andrew Bateman and the Oliver Zangwill Centre (OZC) Clinical Team

(Susan Brentnall, Jon Evans, Donna Gartland, Fergus Gracey, Clare Keohane, Rachel Megoran, Kate Psaila, Barbara Wilson and Giles Yeates)

Oliver Zangwill Centre for Neuropsychological Rehabilitation, Ely, UK

bjective: This cross-sectional descriptive study compares life goals in our outpatient client group with previously published data from another centre which admits clients earlier post-injury, and with more severe impairments. Life goals are interesting because they may be helpful in the process of goal setting, the prioritisation of rehabilitation goals, and designing services to meet user needs. Method: Relative importance of goal areas was identified with the Rivermead Life Goals Questionnaire. Results: 37 clients (age $41+/-17 ; 11$ female) were assessed during the survey period. Median time post injury was 24 months. The frequency with which goals were chosen as extremely important was family 28 , personal care 25 , partner 25 , family life 21 , financial status 15 , contacts 14 , leisure 12 , work 11 , religion 
10. Conclusions: As previous research has shown, people with neurological conditions frequently attach great significance to relationships, personal care and domestic arrangements. Nair et al. reported that work was extremely important to $20 \%$ and not at all important to $50 \%$ of their group of patients with chronic or progressive conditions. In contrast, work was extremely important to $30 \%$ of our group and not at all important to only $16 \%$. Similar shifts in ratings were noted in other domains. An increase in the frequency of 'extremely important' ratings with regard to work, religion or life philosophy is indicative that priorities do vary depending on the setting and time post injury. The findings attest to the utility and face validity of this short questionnaire. Furthermore this research highlights the importance of a range of rehabilitation services to meet the needs of client groups who are at different stages in their recovery.

\title{
Just the Job! Vocational Rehabilitation Within a Neuropsychological Rehabilitation Setting for People With Acquired Brain Injury
}

\author{
Donna Gartland, Sue Brentnall and Rachel Megoran \\ Oliver Zangwill Centre, Ely, UK
}

Qjective: To describe the process of vocational rehabilitation at the Oliver Zangwill Centre. For many clients with acquired brain injury, returning to work is an important goal area, but frequently fraught with difficulties and poor outcomes. This poster explains the process undertaken within The Oliver Zangwill Centre, an interdisciplinary holistic neuropsychological rehabilitation service. It describes the vocational intervention offered, who is involved and the outcomes utilised. Methods: Vocational rehabilitation is organised within a framework for neuropsychological and functional assessment. Steps that we follow include (a) specific data collection to establish work history, interests and current status, (b) formulation of a vocational goal for 6-month rehabilitation program, (c) identification of work skills - general or specific to interests/current role, (d) evaluation of strengths and needs in centre, then in voluntary work experience settings, (e) identify compensatory strategies and modify as needed, (f) formulate vocational action plan and liaise with local services. Conclusions: A case example is described, a man with severe amnesia following subarachnoid haemorrhage, who is now in sustained paid employment following our intervention and liaison with local services. Coordination of input via specialist brain injury services can enable even those people with severe acquired brain injury to return to paid employment.

\section{POSTER SESSION 4: SERVICES AND OUTCOMES}

\section{Profiles of Acquired Brain Injury Clients Presenting to a Community Head Injury Service}

\author{
Noelle O'Keefe
}

Dublin, Ireland

ne hundred and twenty-three individuals with acquired brain injury
attending the services of Headway Ireland - the Irish Association for
Acquired Brain Injury were interviewed using the Head Injury Background
Information Interview schedule. A family member for each client was also
interviewed using the schedule. Figures for the Irish population were collated. 
Figures combine CVA and traumatic acquired brain injuries. In this, a combination of demographics for this client group were reviewed. Personal details, educational and vocational backgrounds, details of injury sustained, hospital attendance, therapy received and subsequent rehabilitation were obtained. Demographic characteristics in relation to acquired brain injury were discussed. Findings from this study were discussed in relation to previous literature. Implications for service provision within an Irish rehabilitation context are discussed.

\title{
Psychological Characteristics of Posttraumatic Headaches and the Effectiveness of Cognitive-Behavioural Therapy for Posttraumatic Headaches
}

\author{
Birgit Gurr and Rudi Coetzer \\ Dorset Healthcare NHS Trust, UK
}

\begin{abstract}
The present study investigated the characteristics of posttraumatic headache, functioning and emotional wellbeing of 41 participants with a traumatic head injury. Self-reported headache disability was compared to a relative's perception of the headache impact. This research also evaluated the effectiveness of a multidimensional cognitive-behavioural approach towards rehabilitation of posttraumatic headaches. This sample included 20 participants with posttraumatic headaches from the original sample of 41. Participants acted as their own controls. Outcome measures included self-rating questionnaires to assess headache severity, intensity, duration, functioning and emotional wellbeing. The headache therapy program proved effective and beneficial. Scores on most of the applied measures improved significantly in comparison to no improvement during a waiting list period.
\end{abstract}

\section{An Exploration of the Experience of Parents Whose Children Sustain Traumatic Brain Injury (TBI) and Their Families}

Alex Clark

University of Plymouth, UK

This study aims to describe and explore the lived experience of parents 1 whose children, aged 0 to 16 years, have sustained a traumatic brain injury (TBI), between two and ten years following injury. The investigation explores the quality of family members' emotional responses to a child's TBI as well as changes in the family's inter-relationships. Links between parents' reported experience and systemic and other psychological theory will be explored with a view to informing rehabilitative practice with children and families. The study employed the qualitative methodology, interpretative phenomenological analysis (IPA), following audiorecording and transcription of semistructured interviews, carried out with 10 parents in community settings. Validity measures include triangulation with quantitative data and independent audit of the analysis. The analysis will generate common themes reflecting experiences shared by the participants as well as discussing individuals' particular experiences. Results of the analysis will be discussed including salient findings relating to parents' emotional responses, relationships and familial interactions following childhood TBI as well as experiences of rehabilitative service provision in South-West England. 


\title{
The Phoenix Unit: A Rehabilitation Project at the Royal Hospital Donnybrook
}

Maeve Nolan and Antonette Owen, on behalf of the multidisciplinary team Royal Hospital, Donnybrook, Ireland

$\mathrm{T}$ This poster presentation outlines a rehabilitation project at the Royal Hospital Donnybrook. The Phoenix Unit was opened in November 2004 to provide a rehabilitation environment in a home-like atmosphere for residents under 65 with acquired brain injury. Seven residents, six with stroke and one with multiple sclerosis, became the project group. The unit sought to provide choice, offer support and encourage personal responsibility using a holistic, patient-centred and goal-directed model of care. It aimed to enhance confidence and maximise autonomy for each resident to the limit of their capabilities. The content of the rehabilitation program together with selection criteria, baseline and follow-up objective and subjective assessment after 3 and 6 months will be described. Residents received input from occupational therapy, physiotherapy, nursing and medical staff. They also had weekly psychosocial meetings to assist them in the transition to the unit. Assessment was carried out by psychology, occupational therapy and social work staff. Residents completed a specially designed questionnaire prior to moving and a follow-up questionnaire after 3 and 6 months. They also completed an Adapted Meyers Lifestyle Questionnaire, a COPE multidimensional coping inventory, a General Health Questionnaire (GHQ) and a Hospital Anxiety and Depression scale (HADS). Results indicate that after 3 months the Phoenix was considered to be beneficial by residents and by staff. In particular, residents welcomed the increase in choice and flexibility in their daily lives, and staff welcomed the improvement in communication between residents and staff. Assessment results indicate that the majority of residents reported an improvement in mood together with improved scores on domestic activities of daily living and decreased anxiety and depression scores. A six-month follow-up will be carried out in May 2005 and results included in the presentation.

\section{Reduced Awareness/Lack of Insight After Traumatic Brain Injury: Considerations for Client Assessment and Service Development}

\begin{abstract}
Alex Concannon
Dublin, Ireland

T has been long established that damage to certain parts of the brain inter1 feres with a person's self-awareness and cognitions (Weinstein and Kahn, 1955). This has implications for the accuracy of self-report of clients with a traumatic brain injury (TBI). This study investigates the range of problems reported by clients with TBI and family members presenting to a community brain-injury rehabilitation service. The sample was 74 consecutive referrals to the service. Both client and relative attended for initial assessment and were interviewed by two members of the rehabilitation team. Client and family members were seen separately and assessed using the Head Injury Problem Schedule (Tyerman 2000), and clients completed the HADS (Zigmond and Snaith 1983). Client and relatives reports were compared using a range of non-parametric tests and variations in reported problems are discussed. The key findings show that relatives reported more cognitive, emotional, social and behavioural problems than clients, and clients who were rated by their relative as having reduced awareness
\end{abstract}


reported significantly fewer cognitive emotional, social and behavioural problems. The implications for the use of client reports to inform the National Physical and Sensory Database and to lead service provision are discussed.

\title{
The Game: Proposal for an Ecological Rehabilitation Program
}

\section{E. Pérez Hernández, S. Fernández Guinea, M. Revenga Sánchez,} and J. González Marqués

Departamento de Psicología Básica Facultad de Psicología Universidad Complutense de Madrid Campus de Somosaguas 28023, Madrid, Spain

\begin{abstract}
$\mathrm{O}$ bjectives: The multidimensional nature of the deficits presented by children referred for neuropsychological rehabilitation requires the development of ecological rehabilitations techniques. When designing a rehabilitation program for children strategies where 'games' are involved should be used because playing is an active form of learning that comprises several cognitive processes. Children permitted to play freely with peers develop skills for seeing things through another person's point of view - cooperating, helping, sharing, and solving problems. The main aim of this study is to develop an ecological rehabilitation program for children with attentional problems, taking this theoretical approach into account. Methods: A novel ecological game-like activity to enhance sustain, selective and set shifting attention has been developed for the purpose of this study. Sixteen 8-year-old children with ADHD will be included in this project. Eight of these children will be trained with the novel ecological method for a period of 3 months ( 1 hour session per week) and the other 8 will be receiving conventional behavioural treatment for the same number of sessions. Attentional skills will be measured before and after the implementation of the rehabilitation program. Results: It is hypothesised that after treatment, children under the ecological game-like program will show better performance on attentional tasks and better generalisation of the learned skills than the group under the conventional behavioural program. Conclusions: One of the main problems in the intervention with children with neuropsychological deficits is their difficulty to generalise what is learned during the clinical sessions to their everyday function. A rehabilitation program that combines daily life activities and games might be helpful in overcoming such difficulties.
\end{abstract}

\section{What is Holistic Neuropsychological Rehabilitation? A Description of the Core Components of the Program at the Oliver Zangwill Centre}

Andrew Bateman and Kate Psaila, with acknowledgement to the entire OZC clinical team (Susan Brentnall, Jon Evans, Donna Gartland, Fergus Gracey, Clare Keohane, Carolyne Machin, Rachel Megoran, Shemin Mohammed, Juliette O'Dell, Barbara Wilson and Giles Yeates)

Oliver Zangwill Centre, Ely, UK

bjective: To describe the 'core components' of the rehabilitation program offered at the Oliver Zangwill Centre. We propose that interdisciplinary formulation represented graphically for feedback to clients is one of the innovative components of the overall approach to holistic rehabilitation. Method: Notes were taken from a series of discussions that were convened to enable review of the mission statement and description of the program. Results: The team revised the mission statement and description 
of the service. Aims of rehabilitation at OZC were stated to be to: enable the client to gain awareness and understanding of the consequences of his/her brain injury, facilitate acceptance and adjustment to the consequences of brain injury; and enable the client to adopt compensatory strategies. It is aimed that therapeutic encounters structured around clients' goals relate to functional activities, social participation and vocational domains. To date, goals have been categorised under the headings of 'understanding brain injury', 'managing activities of daily living', 'recreational activities' and 'work or study plans'. Core components describe how we seek to achieve these aims, through offering a therapeutic milieu, providing opportunity to discover and learn compensatory strategies and skills, involvement of the family in the therapeutic process, where appropriate, providing psychological therapy, drawing on a variety of perspectives including cognitive behavioural therapy, systemic and cognitive-analytic approaches, functional and vocational activities and feedback in the centre and in the client's community, providing frequent repeated contact with an experienced interdisciplinary team that has a shared team understanding of the neuropsychological consequences of brain injury. The clinicians work closely with each other and the client on assessment, formulation and goal planning, therapeutic activities, and reviewing progress. Conclusion: Shared team understanding, is represented by interdisciplinary formulation that includes cognitive, emotional, physical, linguistic, social and functional domains. Understanding is enabled through a range of activities including opportunities to share findings from assessment and goal planning meetings.

\title{
Rehabilitation Guide: A New Assistive Tool for Brain Injury Rehabilitation in Spanish-Speaking Countries
}

\author{
Alvaro Bilbao \\ National Brain Injury Rehabilitation Center, Madrid, Spain
}

Tntroduction: Family information and training is an important part of braininjury-rehabilitation programs worldwide. Beneficial effects of family information and training include better management of physical and cognitive needs of patients, better problem-solving skills in patient care, reduction of caregiver burden, reduction of caregiver stress, and higher quality of life of both patients and families. Although there are over 330 million Spanish speakers worldwide, little or no resources in Spanish have been developed to help family members to better understand and assist in brain injury rehabilitation. Another problem in brain-injury intervention in Spanish-speaking countries is the lack of specialised treatment units in public health systems and economic limitations for private treatment for the majority of the population. Objectives: To present a new tool in Spanish to provide family information on brain injury rehabilitation and review its efficacy in a specific target group. Method: Intra-group differences in quality of life, caregiver burden, knowledge of brain injury rehabilitation, and stress level variables before and after giving the manual were assessed. Participants were 45 family members of brain-injured patients undergoing intensive rehabilitation. Conclusions: The guide has been a useful tool in acquiring more knowledge on brain-injury rehabilitation and reduction of distress. 


\section{A Stroke Awareness Program for Carers}

Maeve Nolan, Patricia Byrne and Karen Smyth on behalf of a multidisciplinary team Royal Hospital, Donnybrook, Ireland

This poster presentation describes an interdisciplinary educational programme for carers of stroke patients at the National Rehabilitation Hospital Dublin. Stroke is a family event and much of the burden of care falls on family members following discharge from hospital. The provision of information and support offered by focused, educational interventions relieves the burden on stroke patient's relatives (Wyller 2003). A Stroke Awareness for Carers program was set up by a team of speech and language therapists, social workers, psychologists, occupational therapists and physiotherapists to provide information and offer support to relatives and carers of inpatients under 65 who were admitted to the hospital following a stroke. It sought to provide information on stroke and its effects, to enable families and carers to gain maximum benefit from rehabilitation, to prepare them for the practical and emotional consequences of providing care after discharge and to emphasise the importance and necessity of self-care for carers. The program consists of a combination of didactic, experiential and supportive group elements. It takes place over two consecutive Fridays for a total of 10 hours, including two lunch breaks. The program includes relevant handouts and booklets and input on the following topics: demystifying the terminology of stroke, coping skills and tips for survival, getting the most from a rehabilitation program, functional and behavioural changes following stroke, accessing entitlements, organising a support network and preparing to manage at home. A key element is a personal experience presentation by a carer. Participants complete a COPE inventory (Carver 1989) and a General Health Questionnaire in order to provide information on health status and common coping strategies of participants. Feedback and evaluation forms are also completed at the end of each programme. Staff and participant feedback indicate that the program achieves its stated objectives. 